\title{
Stochastic Analysis of Reversible Self-Assembly
}

\author{
Urmi Majumder, Sudheer Sahu, John H. Reif \\ Department of Computer Science, \\ Duke University, Durham, NC, USA. \\ \{urmim, sudheer, reif\}@cs.duke.edu
}

\begin{abstract}
The theoretical basis of computational self-assembly dates back to the idea of Wang tiling models in the early 1960s. ${ }^{1}$ More recently, it has been recognized that self-assembly is a promising route to nano-scale computation and there have been many experimental demonstrations of self-assembling DNA tiles performing computation. Winfree ${ }^{2}$ proposed abstract irreversible (only tile accretion is allowed) models for the self-assembly process that can perform universal computation. Realism, however, requires us to develop models and analysis for reversible tiling models, where tile dissociation is also allowed so that we can measure various thermodynamic properties. To date, however, the stochastic analysis of reversible tiling processes has only been done for one-dimensional assemblies and has not been extended to two or three dimensional assemblies. In this paper we discuss how we can extend prior work in one dimension by Adleman et $a .^{3}$ to higher dimensions. We describe how these self-assembly processes can be modeled as rapidly mixing Markov Chains. We characterize chemical equilibrium in the context of self-assembly processes and present a formulation for the equilibrium concentration of various assemblies. Since perfect equilibrium can only be reached in infinite time, we further derive the distribution of error around equilibrium. We present the first known direct derivation of the convergence rates of two and three-dimensional assemblies to equilibrium. Finally we observe that even when errors are allowed in the self-assembly model, the distribution over assemblies converge to uniform distribution with only small number of random association/dissociation events. We conclude with some thoughts on how to relax some of our model constraints.
\end{abstract}

Keywords: self-assembly, rapidly mixing Markov Chains, chemical equilibrium

\section{Introduction}

\subsection{Motivation}

Self-assembly is a ubiquitous process in which small objects self-organize into larger and complex structures. Examples in nature are numerous: atoms self-assemble into molecules, molecules into cells, cells into tissues, and so on. Self-assembly has recently been demonstrated as a powerful technique for constructing nano-scale objects. For example, a wide variety of DNA lattices made from self-assembled branched DNA molecules (DNA tiles) ${ }^{4-10}$ have been successfully constructed (See surveys by $\mathrm{Xu}$ et $a l .{ }^{11}$ and Reif et $a l .{ }^{12}$ ). Peptide self-assembly provides another example of 
nanoscale assembly. ${ }^{13}$ The principle of self-assembly has also been used for mesoscale ${ }^{\mathrm{I}}$ constructions using capillary forces ${ }^{14,15}$ or magnetic forces.

In the context of theoretical self-assembly, mathematical studies of tiling date back to 1960s, when Wang introduced his tiling problem. ${ }^{1}$ The input is a finite set of unit size square tiles, each with a labeled side. The labels are symbols from a finite alphabet. Assuming an arbitrarily large supply of each tile, the problem is to place the tiles, without rotation, to completely fill a given region such that each pair of adjacent sides have identical symbols. The initial focus of research in this area was towards the decidability/undecidability of this tiling problem. ${ }^{17}$ Researchers have also considered the complexity of this problem; for example, Lewis and Papadimitriou proved that the tiling problem for a bounded $n \times n$ region is NP-Complete. A comprehensive study of tiling can be found in the text by Grunbaum et al. ${ }^{18}$ Winfree instigated a revival in the study of tiling in 1996 when he proposed using self-assembling DNA tiles to simulate computation. ${ }^{19}$

\subsection{Theoretical Models of Irreversible Self-Assembly Processes}

In 2000, Rothemund and Winfree ${ }^{20}$ proposed an Abstract Tile Assembly Model (aTAM), which provides a mathematical model for theoretical studies of self-assembly. In this irreversible model, the sides of the tiles are assumed to have the same selective binding constraints (pads) as the Wang tiles, meaning they function as programmable bonds which hold together tiles. The self-assembly process is initiated by a "seed" tile and proceeds to form aggregates by tiles binding together at their pads. This model was later extended by Adleman et al. ${ }^{21}$ to include the time complexity of generating specified assemblies. Later work includes combinatorial optimization, complexity problems, fault tolerance, and topology changes, in the abstract Tile Assembly Model as well as in some of its variants. ${ }^{22,22-42}$

The aTAM provides an elegant framework for programming tiles to form computational assemblies. The resulting assemblies, however, are error-free only because the model assumes a perfect growth. Thus to model various types of errors, Winfree proposed the kinetic Tile Assembly Model $(\mathrm{kTAM})^{43}$ which is based on the physical chemistry of DNA tiles in solution.

Unfortunately one cannot study yields or convergence rates in a kinetic setting. We are not aware of any work giving a thermodynamic characterization of two dimensional (2D) assembly, in the context of a reversible tiling assembly. There has been, however, some chemical kinetics and equilibrium studies in the context of particular and general self-assemblies as discussed below.

\subsection{Study of Chemical Kinetics and Equilibrium in the context of Self-Assembly}

The study of chemical equilibrium has a long history. In determininstic chemical kinetics based on the law of mass action, chemical equilibrium is defined as a limiting state which a system approaches asymptotically. The stochastic theory of chemical reactions alternately defines equilibrium as a stationary distribution over the concentration of reactant and product particles. There is also a mathematical characteristic of system composition that gives a sufficiently sharp indication of the attainment of equilibrium. ${ }^{44}$ Another major theoretical work in this field is the proof for Turing

\footnotetext{
${ }^{\mathrm{I}}$ It is the size scale between nanoscale and micron scale.
} 
Universality of chemical kinetics. ${ }^{45}$ A good summary of the various stochastic approaches and applications to chemical reaction kinetics can be found in. ${ }^{46}$

In the context of protein self-assembly, researchers have studied the various mechanisms by which a linear or helical polymer could assemble. ${ }^{47}$ Beyond linear assemblies, Israelachvili proposed general thermodynamic constraints regarding the growth of multidimensional assemblies. ${ }^{48}$ In two dimensions, macroscopic aggregates grow abruptly once the concentration reaches a critical level (nucleation). It has been concluded that nucleation is usually associated with phase transitions and hence can be described with familiar thermodynamic models. ${ }^{49}$ Besides protein assembly, examples of more general models include the model proposed by Ecrolani ${ }^{50}$ where the growth of desired complexes in competition with non-linear random polymerization was studied. There was, however, no work on tiling assemblies until Adleman et al. ${ }^{3}$ proposed a stochastic model for linear tiling assemblies as discussed in the following subsection.

\subsection{Prior Work on Stochastic Analysis of Linear Assemblies}

From the discussion in Subsection 1.2, it is quite evident that an abstract model for reversible tile assembly would be very useful. In particular, determining thermodynamic properties such as equilibrium concentration and convergence rates under the reversible tiling model can be used to predict yields of different computational self-assembling systems. Recall that while equilibrium concentration informs us about the amount of substrate required to guarantee a certain yield, convergence rates directly measure the time complexity of the computation.

Adleman et $a l^{3}{ }^{3}$ initiated this study with his analysis of the properties of reversible linear assemblies. They assumed that there are $n$ types of tiles arranged on a doubly infinite line. Each tile type is assigned a probability function and those density functions sum up to 1 . Some of the tiles may be bonded to their neighbors. At every time step, all the tiles are scrambled so that they fall at random positions on the infinite line without overlap. Next an existing bond is broken with an "off" probability and two free ends of adjacent tiles bond with a given "on" probability. Adleman et al. ${ }^{3}$ investigated whether such linear assemblies can attain equilibrium. They characterized the equilibria for linear self-assembling systems and discussed convergence rates of these assemblies to equilibria. Characterization of equilibria and measuring convergence rates in the context of reversible general $2 \mathrm{D}$ tiling systems, however, remained an open question. ${ }^{51}$

\subsection{Rapidly Mixing Markov Chains for Physical Models}

A Rapidly Mixing Markov Chain is a Markov Chain that converges to its stationary distribution in time polynomial in the length of the state space. One of the main goals of this paper is to investigate if self-assembly can be modeled as rapidly mixing Markov Chains (rmMC). Selfassembly processes have often been described as continuous time Markov Chains. ${ }^{43}$ Researchers have studied other physical processes as rmMC. Some example systems which have been widely studied are monomer-dimer systems (e.g. theory of mixtures of molecules of different sizes), ${ }^{52}$ cell cluster theory of the liquid state ${ }^{53}$ and the model for the adsorption of diatomic molecules on a crystal surface. ${ }^{54}$ Some other models for which there exist interesting mixing time results are the hard core model, ${ }^{55}$ the Ising Model ${ }^{56}$ six-point ice model ${ }^{57}$ and the self-avoiding walk model 
for linear polymers. ${ }^{58,59}$ Randall and others ${ }^{60-62}$ have extensively studied several rapidly mixing Markov Chains for random tiling with applications in both physics and computer science and have proved that in many interesting cases, a small number of random moves suffice to generate a uniform distribution over tilings. Thus they show that some of these chains are rapidly mixing. They, however, used indirect proof techniques for limited parameter settings rather than direct proof techniques for general parameter settings.

\subsection{Prior Work on Convergence Rate Analysis of Two and Three Dimensional Assemblies}

In 1995, Luby et al. ${ }^{63}$ proposed several Markov Chain algorithms for planar lattice structures. They presented techniques which proved that a small number of random moves suffice to obtain a uniform distribution over the configuration space of lozenge tiling and domino tiling. A domino tiling is a covering of a finite region of the Cartesian lattice with dominoes, where each domino covers two adjacent squares of the region. A lozenge tiling is the analogue of a domino in the Cartesian lattice; each lozenge covers two adjacent triangles in the triangular lattice, and has three possible orientations. It can be easily seen that lozenge tiling and $3 \mathrm{D}$ self-assembled lattices are equivalent. The rapid mixing for the Markov chain for lozenge tiling can be proved using a coupling argument on the lozenge routing, a set of non-intersecting shortest paths on the Cartesian lattice. Further it can be easily proved that the case of single path routing can be applied to show that $2 \mathrm{D}$ self-assembly is rapidly mixing.

In this paper not only we present a more direct derivation of the convergence rates of two and three dimensional assemblies to equilibrium but also characterize chemical equilibrium in the context of self-assembly process and present a formulation for the equilibrium concentration of various assemblies. Since perfect equilibrium can only be reached in infinite time, we further derive the distribution of error around equilibrium. Finally we observe that even when errors are allowed in the self-assembly model, the distribution over assemblies converge to uniform distribution with only a small number of random association/dissociation events.

\subsection{Organization of the Paper}

Since the general problem is intractable, ${ }^{51}$ we address an important subclass of the $2 \mathrm{D}$ system. We first characterize equilibrium in two dimensions (2D) in Section 2. This section builds on Adleman et al.' $\mathrm{s}^{3}$ work on linear assemblies but also relies on the concept of bond pair equilibrium. Section 2 proves that every 2D assembly eventually goes to equilibrium. In Section 3 we draw the correlation between Markov Chains and self-assembling systems. In particular, we prove that certain instances of self-assembly processes with significant importance in nano-assembly, can be viewed as a rapidly mixing Markov Chain ${ }^{\mathrm{II}}$ with appropriate parameter setting. In Section 4 we characterize equilibrium of a $n \times n$ completely addressable square and prove that the corresponding convergence rate decays exponentially in time. In Sections 4, 5 and 6 we extend the equilibrium characterization and derivation of convergence rate of a $n \times n$ completely addressable square to

\footnotetext{
${ }^{\mathrm{II}}$ Rapidly Mixing Markov Chain is a Markov Chain with a polytime (polynomial in the number of tiles.) convergence to its stationary distribution
} 
both general shapes in two dimensions and 2D algorithmic assemblies. Since perfect equilibrium is only attained in infinite time, we derive the probability distribution of error for a small linear perturbation from the thermodynamic equilibrium for computational assemblies in Section 6 . In Section 7 , we further extend the stochastic analysis to a simple three-dimensional $n \times n \times n$ cube. In Section 8 we shift our attention to erroneous assemblies where the error is due to partial mismatch. We observe that, even in the 1D case, approximate equilibrium is reached in time polynomial in the number of tiles. We prove that similar behavior can be observed for two and three dimensions as well. In Section 9 we conclude the paper with some remaining open questions.

\section{Reversible 2D Assemblies by Monomer Addition}

\subsection{The Model}

The main assumptions in our model of 2D assembly processes are as follows: (1) Assembly starts with a pre-assembled nucleating assembly which we call the boundary III, (2) We allow only monomer association and dissociation from a supertile ${ }^{\mathrm{IV}}$, (3) A tile can attach to a growing face of a supertile only where it has at least two correct neighbors. Thus this is an error-free model, (4) A tile can dissociate from a growing face of an assembly only where it has at most two neighbors. This assumption implies that the assembly is always concave. Further, the preassembled boundary does not dissociate at any time during the whole process, (5) All bonds apart from those in the pre-assembled boundary are of the same strength, (6) All tiles have the same probability of association (on probability) and dissociation (off probability), (7) The tiles are not allowed to be rotated.

\subsection{Equilibrium Characterization}

We assume that there are $\mathrm{n}$ types of tiles $T_{1}, \ldots T_{n}$ arranged in a fixed sized region of a twodimensional plane, with $\alpha_{i}$ being the relative fraction of tiles of type $i$.

$$
\begin{aligned}
\alpha_{i} & >0 \\
\sum_{i} \alpha_{i} & =1
\end{aligned}
$$

Let $a_{i j}$ denote the fraction of $T_{i}$ tiles that are bonded to a $T_{j}$ tile on the right. Let $b_{i k}$ be the fraction of $T_{i}$ tiles that are bonded to a $T_{k}$ tile on the top. Let $p_{i j}$ be the fraction of $T_{j}$ tiles that are bonded to a $T_{i}$ tile on left, and let $q_{i k}$ be the fraction of tiles of type $T_{k}$ that are bonded to a $T_{i}$ type of tile at the bottom. Define $a_{i}$ to be fraction of $T_{i}$ that is free at right, $b_{i}$ as a fraction of $T_{i}$ that is free at top, $p_{j}$ as a fraction of $T_{j}$ free at left, $q_{k}$ as fraction of $T_{k}$ free at bottom. Thus,

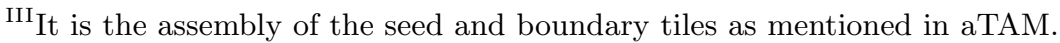

${ }^{\mathrm{IV}} \mathrm{A}$ supertile is maximal contiguous set of tiles that are bonded together.
} 


$$
\begin{aligned}
\sum_{j} a_{i j}+a_{i} & =1 \\
\sum_{k} b_{i k}+b_{i} & =1 \\
\sum_{i} p_{i j}+p_{j} & =1 \\
\sum_{i} q_{i k}+q_{k} & =1
\end{aligned}
$$

Define

$$
\begin{aligned}
A_{i j} & =\alpha_{i} a_{i j} \\
B_{i k} & =\alpha_{i} b_{i k} \\
P_{i j} & =\alpha_{j} p_{i j} \\
Q_{i k} & =\alpha_{k} q_{i k}
\end{aligned}
$$

Clearly,

$$
\begin{aligned}
\sum_{j} A_{i j}+A_{i} & =\alpha_{i} \\
\sum_{k} B_{i k}+B_{i} & =\alpha_{i} \\
\sum_{i} P_{i j}+P_{j} & =\alpha_{j} \\
\sum_{i} Q_{i k}+Q_{k} & =\alpha_{k}
\end{aligned}
$$

$A_{i j}$ can be interpreted as the fraction of neighboring pairs of tiles such that the left tile is of type $T_{i}$, and right tile be of type $T_{j}$, and there is bond between the two. $A_{i}$ is the fraction of tiles of type $T_{i}$ and free to the right. Clearly,

$$
\begin{aligned}
A_{i j} & =P_{i j} \\
\sum_{i} A_{i} & =\sum_{j} P_{j}
\end{aligned}
$$

Similarly,

$$
\sum_{i} B_{i}=\sum_{k} Q_{k}
$$


Further define

$$
\begin{aligned}
& F_{R}=\sum_{i} A_{i} \\
& F_{T}=\sum_{i} B_{i}
\end{aligned}
$$

We define bond-pair equilibrium as an equilibrium when for every tile of type $T_{j}$, the total number $\left(C_{i j k}\right)$ of $\left(A_{i j}, B_{k j}\right)$ in the assembly is invariant over time steps. Informally independence means bond forming on one side of the tile cannot affect what bonds can form on the other three sides.

Theorem 2.2.1 Bond pair equilibrium and independence implies strong equilibrium.

Proof In our model, we consider monomeric addition and dissociation at the growing face of the assembly. During a step of the assembly a tile $T_{j}$ having only two neighbors (left $T_{i}$ and bottom $T_{k}$ ) breaks the two bonds with probability $\tau_{i j}$ and $\tau_{k j}^{\prime}$. Thus the probability that $T_{j}$ leaves this growth site by breaking the bonds with $T_{i}$ and $T_{k}$ is $\sum_{k}\left(A_{i j} \cdot B_{k j} \cdot A_{j} \cdot B_{j} \cdot \tau_{i j} \cdot \tau_{k j}^{\prime}\right)$ $=A_{i j} \cdot A_{j} \cdot B_{j} \cdot \tau_{i j} \cdot \sum_{k}\left(B_{k j} \cdot \tau_{k j}^{\prime}\right)$.

A tile $T_{j}$ can come and attach at a location on growing face where it will form bonds with $T_{i}$ to left and $T_{k}$ to south. On probabilities for these bonds will be $\sigma_{i j}$ and $\sigma_{k j}^{\prime}$. Hence, the probability that $T_{j}$ attaches to this growth site by binding with $T_{i}$ and $T_{k}$ is $\sum_{k}\left(A_{i} \cdot B_{k} \cdot \frac{P_{j}}{F_{R}} \cdot \frac{Q_{j}}{F_{T}} \sigma_{i j} \sigma_{k j}^{\prime}\right)=$ $A_{i} \cdot \frac{P_{j}}{F_{R}} \cdot \frac{Q_{j}}{F_{T}} \sigma_{i j} \sum_{k}\left(B_{k} \cdot \sigma_{k j}^{\prime}\right)$

Therefore, the overall update rule is,

$$
\Delta A_{i j}=-A_{i j} \cdot A_{j} \cdot B_{j} \cdot \tau_{i j} \cdot \sum_{k} B_{k j} \cdot \tau_{k j}^{\prime}+A_{i} \cdot \frac{P_{j}}{F_{R}} \cdot \frac{Q_{j}}{F_{T}} \sigma_{i j} \sum_{k} B_{k} \cdot \sigma_{k j}^{\prime}
$$

For bond equilibrium,

$$
\Delta A_{i j}=0
$$

Thus,

$$
\frac{\sigma_{i j} \sum_{k} B_{k} \cdot \sigma_{k j}^{\prime}}{\tau_{i j} \cdot \sum_{k} B_{k j} \cdot \tau_{k j}^{\prime}}=\frac{A_{i j} A_{j} B_{j} F_{R} F_{T}}{A_{i} \cdot P_{j} \cdot Q_{j}}
$$

But this not enough in the context of $2 \mathrm{D}$ assemblies. On the other hand, in case of bond-pair equilibrium,

$$
\Delta C_{i j k}=0
$$


Hence,

$$
\begin{aligned}
-A_{i j} \cdot A_{j} \cdot B_{j} \cdot \tau_{i j} \cdot B_{k j} \cdot \tau_{k j}^{\prime}+A_{i} \cdot \frac{P_{j}}{F_{R}} \cdot \frac{Q_{j}}{F_{T}} \sigma_{i j} B_{k} \cdot \sigma_{k j}^{\prime} & =0 \\
\frac{\sigma_{i j} B_{k} \cdot \sigma_{k j}^{\prime}}{\tau_{i j} \cdot B_{k j} \cdot \tau_{k j}^{\prime}} & =\frac{A_{i j} A_{j} B_{j} F_{R} F_{T}}{A_{i} \cdot P_{j} \cdot Q_{j}}
\end{aligned}
$$

Recall that a supertile is a maximal contiguous set of tiles that are bonded together. Further in our model we assume that the boundary row and column are pre-assembled. Let us assume that the fraction of pre-assembled boundary is $\{$ Boundary $\}$. Now, consider a supertile $\Gamma$, as shown in Figure 1. Assume that $\{\Gamma\}$ denotes the fraction of supertiles that are of type $\Gamma$. Assuming independence, $\{\Gamma\}=\{$ Boundary $\} \cdot a_{\text {Boundary }\left(r_{1}\right) i_{1}} \cdot b_{\text {Boundary }\left(c_{1}\right) i_{1}} \cdot a_{\text {Boundary }\left(r_{2}\right) i_{2}} \cdot b_{i_{1} i_{2}} \cdots \cdots a_{i^{\prime} i} \cdot a_{i} \cdot b_{k}$.

Now consider the addition of monomer tile $T_{j}$ (or call it supertile of type $T_{j}$ ) to $\{\Gamma\}$ to form another supertile $\Gamma \cdot T_{j}$ as shown in Figure 1. Assuming independence,

$$
\begin{aligned}
\left\{\Gamma \cdot T_{j}\right\}= & \{\text { Boundary }\} \cdot a_{\text {Boundary }\left(r_{1}\right) i_{1}} \cdot b_{\text {Boundary }\left(c_{1}\right) i_{1}} \cdot a_{\text {Boundary }\left(r_{2}\right) i_{2}} \cdot b_{i_{1} i_{2}} \\
& \cdots \cdots a_{i^{\prime} i} \cdot a_{i j} \cdot b_{k j} \cdot a_{j} \cdot b_{j}
\end{aligned}
$$

Since $T_{j}$ is a monomer, it is unbounded from all sides, we get the following assuming independence:

$$
\left\{T_{j}\right\}=\frac{p_{j}}{F_{R}} \cdot \frac{q_{j}}{F_{T}} \cdot a_{j} \cdot b_{j}
$$

Hence

$$
\begin{aligned}
\frac{\left\{\Gamma \cdot T_{j}\right\}}{\{\Gamma\}\left\{T_{j}\right\}} & =\frac{a_{i j} \cdot b_{k j} \cdot a_{j} \cdot b_{j} \cdot F_{R} \cdot F_{T}}{a_{i} \cdot b_{k} p_{j} \cdot q_{j} \cdot a_{j} \cdot b_{j}} \\
& =\frac{a_{i j} \cdot F_{R} \cdot F_{T}}{a_{i} \cdot p_{j} \cdot q_{j}} \cdot \frac{b_{k j}}{b_{k}}
\end{aligned}
$$

Assuming bond-equilibrium,

$$
\frac{\left\{\Gamma \cdot T_{j}\right\}}{\{\Gamma\}\left\{T_{j}\right\}}=\frac{\sigma_{i j} \sum_{k} B_{k} \cdot \sigma_{k j}^{\prime}}{\tau_{i j} \cdot \sum_{k} B_{k j} \cdot \tau_{k j}^{\prime}} \cdot \frac{B_{k j}}{B_{k}}
$$

However, if we assume bond-pair equilibrium, then we have

$$
\begin{aligned}
\frac{\left\{\Gamma \cdot T_{j}\right\}}{\{\Gamma\}\left\{T_{j}\right\}} & =\frac{\sigma_{i j} B_{k} \cdot \sigma_{k j}^{\prime}}{\tau_{i j} \cdot B_{k j} \cdot \tau_{k j}^{\prime}} \cdot \frac{B_{k j}}{B_{k}} \\
& =\frac{\sigma_{i j} \cdot \sigma_{k j}^{\prime}}{\tau_{i j} \cdot \tau_{k j}^{\prime}}
\end{aligned}
$$

which implies strong equilibrium. 


\subsection{Properties}

In this section we prove that every $2 \mathrm{D}$ assembly which grows by monomer addition reaches equilibrium. The uniqueness of the equilibrium depends on the particular instance of the problem.

As in Adleman et al. ${ }^{3}$ we will demonstrate in this section that equilibrium in two dimensions is reached when the entropy density of the $2 \mathrm{D}$ assembly is maximized. Recall that entropy density was the sum of the internal entropy density and external entropy density.

Theorem 2.3.1 Every 2D assembly goes to equilibrium, defined by the maximum entropy density.

Proof Internal entropy density $S_{I}$ of a $2 \mathrm{D}$ assembly is defined to be the information theoretic entropy of the triple $\left(T(x, y), B_{x}(x), B_{y}(y)\right)$ where $T(x, y)$ denotes the tile at location $T(x, y)$ while $C_{x}(x)=1$ denotes that $T(x, y)$ is bonded to its left neighbor $T(x-1, y)$ and $C_{y}(y)=1$ denotes that $T(x, y)$ is bonded to its bottom neighbor $T(x, y-1)$. By the independence assumption,

$$
\begin{array}{r}
S_{I}=H\left[T(x, y), C_{x}(x), C_{y}(y) \mid T(x, y-1), T(x-1, y)\right] \\
=\sum_{i} \alpha_{i} \sum_{j} \alpha_{j} H\left[T(x, y), C_{x}(x), C_{y}(y) \mid T(x, y-1)\right. \\
\left.=T_{j}, T(x-1, y)=T_{i}\right]
\end{array}
$$

where $H$ is the information-theoretic entropy of the discrete random variable $\left(T(x, y), C_{x}(x), C_{y}(y)\right)$.

Suppose that the tile at position $(x-1, y)$ is a $T_{i}$, the tile at position $(x, y-1)$ is a $T_{j}$ and the tile at position $(x, y)$ is $T_{k}$. Now we have four cases to consider:

1. there can be a bond between $T_{i}$ and $T_{k}$ as well as $T_{j}$ and $T_{k}$

2. there is no bond between either $T_{i}$ and $T_{k}$ or $T_{j}$ and $T_{k}$

3. there is a bond between $T_{i}$ and $T_{k}$ but there is no bond between $T_{j}$ and $T_{k}$

4. there is a bond between $T_{j}$ and $T_{k}$ but there is no bond between $T_{i}$ and $T_{k}$

Consequently,

$$
\begin{aligned}
H\left[T(x, y), C_{x}(x), C_{y}(y) \mid\right. & \\
\left.T(x, y-1)=T_{j}, T(x-1, y)=T_{i}\right]= & -\left(\sum_{k} a_{i k} b_{j k} \ln a_{i k} b_{j k}\right. \\
& +\sum_{k} a_{i} b_{j} \frac{P_{k}}{F_{R}} \frac{Q_{k}}{F_{T}} \ln a_{i} b_{j} \frac{P_{k}}{F_{R}} \frac{Q_{k}}{F_{T}} \\
& +\sum_{k} a_{i k} b_{j} \frac{Q_{k}}{F_{T}} \ln a_{i k} b_{j} \frac{Q_{k}}{F_{T}} \\
& \left.+\sum_{k} b_{j k} a_{i} \frac{P_{k}}{F_{R}} \ln b_{j k} a_{i} \frac{P_{k}}{F_{R}}\right)
\end{aligned}
$$


Thus,

$$
\begin{aligned}
S_{I}= & -\sum_{i} \alpha_{i} \sum_{j} \alpha_{j}\left(\sum_{k} a_{i k} b_{j k} \ln a_{i k} b_{j k}+\sum_{k} a_{i} b_{j} \frac{P_{k}}{F_{R}} \frac{Q_{k}}{F_{T}} \ln a_{i} b_{j} \frac{P_{k}}{F_{R}} \frac{Q_{k}}{F_{T}}+\right. \\
& \left.\sum_{k} a_{i k} b_{j} \frac{Q_{k}}{F_{T}} \ln a_{i k} b_{j} \frac{Q_{k}}{F_{T}}+\sum_{k} b_{j k} a_{i} \frac{P_{k}}{F_{R}} \ln b_{j k} a_{i} \frac{P_{k}}{F_{R}}\right)
\end{aligned}
$$

Simplifying this expression, we obtain

$$
\begin{aligned}
S_{I}= & \sum_{i} \sum_{j} \alpha_{i} \alpha_{j} \ln \alpha_{i} \alpha_{j}+\sum_{i} \sum_{j} A_{i} B_{j} \ln \frac{1}{A_{i} B_{j}}+\sum_{k} \sum_{k} P_{k} Q_{k} \ln \frac{1}{P_{k} Q_{k}}+ \\
& F_{R} F_{T} \ln F_{R} F_{T}+\sum_{i k} \sum_{j k} A_{i k} B_{j k} \ln A_{i k} B_{j k} .
\end{aligned}
$$

Define the external entropy as

$$
S_{X}=\sum_{i k} \sum_{j k} A_{i k} B_{j k} \ln \frac{\sigma_{i k}}{\tau_{i k}} \frac{\sigma_{j k}}{\tau_{j k}}
$$

and entropy density as

$$
S=S_{I}+S_{X}
$$

Observe that

$$
\frac{\partial^{2} S}{\partial A_{i k} \partial B_{j k}}=0 \quad \text { iff } \quad \frac{A_{i k} B_{i k} A_{k} B_{k} F_{R} F_{T}}{A_{i} B_{j} P_{k} Q_{k}}=\frac{\sigma_{i k} \sigma j k}{\tau_{i k} \tau_{j k}}
$$

Further for any vector $\left(\left(v_{12}, u_{12}\right), \ldots\left(v_{n-1, n}, u_{n-1, n}\right)\right)$

$$
\sum_{v_{i k}, v_{x y}} \sum_{u_{j k}, u_{y z}} \frac{\partial^{4} S}{\partial A_{i k} \partial A_{x y} \partial B_{j k} \partial B_{y z}} v_{i k} v_{x y} u_{j k} u_{y z} \leq 0
$$

and the equality holds only when $\left(\left(v_{12}, u_{12}\right), \ldots\left(v_{n-1, n}, u_{n-1, n}\right)\right)$ is a zero vector.

Moreover, for some bond $(i, k)$ along the $\mathrm{x}$ axis and $(j, k)$ along the $\mathrm{y}$ axis

$$
\sum \frac{\partial^{2} S}{\partial A_{i k} \partial B_{j k}} \frac{\partial A_{i k} \partial B_{j k}}{\partial^{2} t}>0 \quad \text { for } \quad \frac{\partial A_{i k} \partial B_{j k}}{\partial^{2} t} \neq 0
$$

and for all $i, j, k$.

$$
\sum \frac{\partial^{2} S}{\partial A_{i k} \partial B_{j k}} \frac{\partial A_{i k} \partial B_{j k}}{\partial^{2} t}=0 \quad \text { when } \quad \frac{\partial A_{i k} \partial B_{j k}}{\partial^{2} t}=0
$$

Recall that bond pair equilibrium and independence imply strong equilibrium. All the above imply 
that strong equilibrium is reached when total entropy densty is maximized and total entropy density of a $2 \mathrm{D}$ assembly increases until bond pair equilibrium ${ }^{\mathrm{V}}$.

\section{Self-Assembly Processes as Rapidly Mixing Markov Chains}

Observe that we can define a discrete time Markov Chain (MC) for some finite assemblies such as those modeled in this paper with an exponential state space ${ }^{\mathrm{VI}}$. We define a transition as either a single instance of one subassembly combining with another (possibly different) instance of subassembly to form a larger assembly or the dissociation of an assembly into two subassemblies.

\subsection{Existence of stationary distribution}

Theorem 3.1.1 The MC for a finite reversible self-assembly process has a stationary distribution.

Proof For a MC to have a stationary distribution it must satisfy the properties of finiteness, irreducibility, aperiodicity and positive recurrence. Observe that the chain is finite since we have finite number of tiles. Hence the number of possible configurations is also finite. The chain is also irreducible (or connected) since any state can be reached from any other state by a series of transitions. It is also aperiodic, since every state has a non-zero probability of remaining in the same state. The property of positive recurrence follows from the finite state space.

\subsection{Correlation between Chemical Equilibrium and Stationary States}

Theorem 3.2.1 An assembly in chemical equilibrium implies that the corresponding Markov Chain has reached its ergodic limit, but the reverse is not always true.

Proof An assembly in chemical equilibrium has equal forward and backward reaction rates. Hence the net concentration change of reactants and products is zero. Thus, the concentration of each component remains constant, corresponding with the steady state. However, when the MC has a stationary distribution, the rate of change of all the components in the reaction system may be zero but that does not imply that forward and backward reaction rates are equal.

\subsection{Rapidly Mixing Markov Chain Interpretation of Self-Assembly Processes}

\subsubsection{Definition}

The time required for a Markov Chain to converge to its stationary distribution is also known as the mixing time of the chain. It is measured in terms of the total variation distance between the distribution at time $\mathrm{t}$ and the stationary distribution. Thus if $\Omega$ denotes the state space and if $P^{t}(x, y)$ is the probability for going from $x$ to $y$ in $t$ steps, the total variation distance at time $t$ is defined as

$$
\left\|P^{t}, \pi\right\|=\max _{x \in \Omega} \frac{1}{2} \sum_{y \in \Omega}\left|P^{t}(x, y)-\pi(y)\right|
$$

\footnotetext{
${ }^{\mathrm{V}}$ Although we derive the critical points for a continuous system in this section, for the analysis of the Markov Chains in the following sections we will consider the corresponding discrete systems.

${ }^{\mathrm{VI}}$ If we represent individual tiles as nodes in a graph, where edges represent bonding, then we can represent all possible sub-assemblies of an assembly of size $n$ by the presence/absence of edge between appropriate vertices, yielding $O\left(2^{n^{2}}\right)$ possible states
} 
This is just the $L_{1}$ norm with the $\frac{1}{2}$ introduced so that the distance is always at most 1 . For $\epsilon>0$, the mixing time $\tau(\epsilon)$ is given as $\tau(\epsilon)=\min \left\{t:\left\|P^{t^{\prime}}, \pi\right\| \leq \epsilon, \forall t^{\prime} \geq t\right\}$. A Markov Chain for a tiling self-assembly is rapidly mixing if the mixing time is bounded by a polynomial in $n$ and $\log \epsilon^{-1}$ where $n$ is the length of each tiling in the state space.

\subsubsection{Importance}

In the context of self-assembly processes, proving that a particular assembly process can be represented as a rapidly mixing Markov Chain has far reaching consequences. First it gives a better understanding of the thermodynamics of the assembly process, in particular whether the equilibrium can be attained in polynomial time. In the context of self-healing assemblies, this information is useful in estimating the repair speed of a damaged lattice. We will use the example of a reversible $n$-linear polymerization in one dimension ${ }^{3}$ to demonstrate how we can correlate self-assembly processes and rapidly mixing Markov Chains. In the following sections we will see some 2D and 3D examples of self-assemblies for which the corresponding Markov Chain is rapidly mixing. VII

\subsection{3 n-linear Polymerization}

In $n$-linear polymerization, there are unlimited copies of the tiles $T_{1}, T_{2}, \ldots, T_{n}$ and the assembly rule is that the right end of $T_{i}$ is attached to the left end of $T_{i+1}, \forall i=1, \ldots, n-1$ and no other attachment is possible.

For reversible polymerization, Adleman et al. ${ }^{3}$ analyzed the time at which the fraction of free gluable surfaces, goes to a fixed point. Specifically he showed that the fraction is guaranteed to be within $\epsilon$ distance from the equilibrium value $\beta$ if time is at least $\max \left\{3, \frac{1}{r}\right\}\left(\ln \frac{1}{\epsilon}\right)$ where $r$ is the off probability. They also showed that a unique equilibrium is reached irrespective of the initial state.

Theorem 3.3.1 The Markov Chain for reversible n-linear polymerization is rapidly mixing.

Proof By the results of Adleman et al. ${ }^{3}$ the chemical equilibrium for reversible, $n$-linear polymerization is attained in $O\left(\log \frac{1}{\epsilon}\right)$. Further, using theorem 3.2.1, we can show that the corresponding Markov Chain reaches a unique steady state in time which is a function of just $\log \frac{1}{\epsilon}$. Hence, it is rapidly mixing.

Later sections discuss the mixing times for MCs in two and three dimensions.

\section{Assembly of $n \times n$ Completely Addressable Square}

In $n \times n$ completely addressable square there are "sufficient" copies VIII of tiles $T_{1,1}, T_{1,2}, \ldots, T_{1, n}$, $T_{2,1}, \ldots, T_{2, n}, \ldots, T_{n, 1}, \ldots, T_{n, n}$ and the assembly rule is that the right side of tile $T_{i, j}$ attaches with the left side of $T_{i+1, j}$ while the top pad of $T_{i j}$ binds to the bottom pad of tile $T_{i, j+1}$ for all $i, j \mathrm{~s}$ in $\{1,2, \ldots, n-1\}$. Further the bottom pad of tile $T_{i, 1}$ binds to top pad of boundary tile $c_{i}$ for all $i \in\{1,2, n\}$ while the left pad of $T_{1, j}$ binds to the right pad of boundary tile $r_{j}$ for all

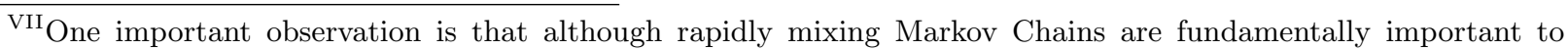
understand self-assembly computations, our goal is not to solve NP-Complete search problem that require exponential volume, to try all possibilities. Hence this is not considered in this paper.

VIII Thus for the assembly, we will have enough monomers yet state space of the corresponding Markov Chain will still be finite.
} 
$i \in\{1,2, n\}$ as shown in figure 1 . We study the behavior of self-assembling a completely addressable $n \times n$ square under the reversible model. Our system comprises of $n^{2}$ different tile types, a set of their corresponding fractions and a pair of on/off probabilities, assumed to be equal for every binding/dissociation of two tiles. Thus all allowable bonds have the same on-rate $\sigma \in[0,1]$ and off-rate $\tau \in[0,1]$.

Recall that we assume that all boundary tiles have already assembled and if we assume that the glue strengths between any two pads is 1 , a tile will attach only to the site where it will have 2 neighbors. Hence the size of the assembled structure is $(n+1) \times(n+1)$. The portion of interest is of size $n \times n$.

\subsection{Equilibrium Characterization}

For the sake of simplicity we assume that a tile will bond to a position where it will have a bottom and a left neighbor. We also assume that an already attached tile can dissociate only from a position, where it has not more two neighbors. For example, in the Figure 1 the shown aggregate has one of its binding sites at the marked position. In general, the assembly grows in the north-east direction.

Let $T_{i, j}$ denote the tile type that can attach at position $(i, j)$ in the aggregate, which is the $i^{t h}$ position from bottom and $j^{\text {th }}$ position from left. We assume that tiles of type $T_{i, j}$ can attach only to locations where it will have a left neighbor and a bottom neighbor.

Theorem 4.1.1 The assembly of an $n \times n$ completely addressable square achieves strong equilibrium when each $T_{i, j}$ is free on both top and right pads with probability $1-\frac{\sigma}{\tau n^{2}}$.

Proof Suppose that $a_{i, j}(t)$ be the fraction of tiles of type $T_{i, j}$ that are free at the bottom and $b_{i, j}(t)$ be the fraction of tiles of type $T_{i, j}$ that are free in the left. Since we assume same on probability $\sigma$ for both top and right pads and same off probability $\tau$ for the same $a_{i, j}(t)=b_{i, j}(t)$. Assume for now that $a_{i j}$ assumes a common value that is approximately $a$, for all $i, j$ 's. We will show later [Theorem 4.3.1], that $a_{i, j}$ for all $i, j$ 's converges to a common value $a$ exponentially fast.

We estimate the values of $a$ after 1 step. The update rule in this case would be similar to that in equation 2.2.20: IX $^{\text {IX }}$

$$
a^{\prime}-a=(1-a) \cdot a \cdot a \cdot \tau \cdot(1-a) \cdot \tau-a \cdot \frac{a}{n^{2} a} \cdot \frac{a}{n^{2} a} \cdot \sigma \cdot a \cdot \sigma
$$

Observe that this transformation has a unique fixed point in the interval $[0,1]$ given by $\beta=$ $1-\frac{\sigma}{\tau n^{2}}$. In other words when $a=\beta$, the system is in bond pair equilibrium and each $T_{i j}$ is free on its top and right with an independent probability $\beta$ respectively. Hence, by Theorem 2.2.1, this implies that the tile system has a strong equilibrium.

\footnotetext{
${ }^{\mathrm{IX}}$ Note that we are computing the complement of the variable whose update rule was determined in equation 2.2.20. Besides we use simply bond equilibrium instead of bond pair equilibrium. This is acceptable since for completely addressable structures update rules assuming either bond equilibrium or bond pair equilibrium yield the same result.
} 


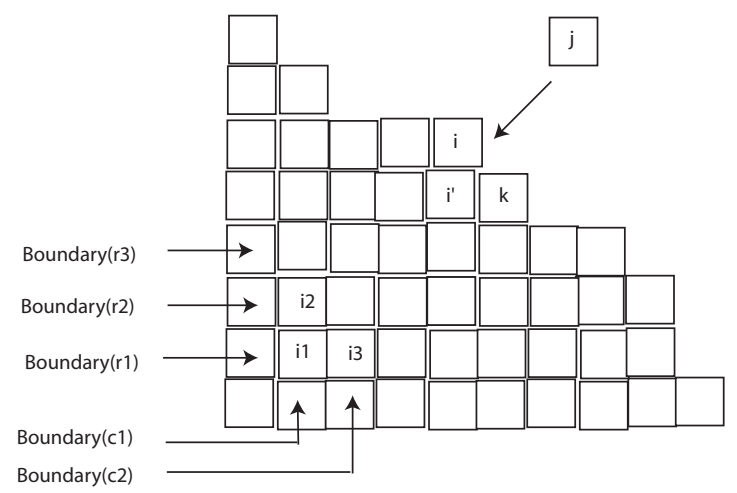

Figure 1: A supertile $\Gamma$ during an assembly.

\subsection{Derivation of Convergence Rates}

Let $\Delta(t)$ denote the distance of $a(t)$ from $\beta$ i.e. $\Delta(t)=a(t)-\beta$.

Theorem 4.2.1 In the reversible assembly of an $n \times n$ completely addressable square, $\Delta(t)$ decays exponentially in $t$ for $n \geq 3$. In particular, time taken $t$ to reach $\epsilon$ distance from equilibrium is given by $\frac{\log \frac{1-\beta}{\epsilon}}{\log \frac{1}{c_{2}}}<t<\frac{\log \frac{1-\beta}{\epsilon}}{\log \frac{1}{c_{1}}}$ where $\beta$ is the equilibrium value of a and $c_{1}=1-\tau \frac{\sigma}{n^{2}}$ and $c_{2}=1-\tau\left(6 \frac{\sigma}{n^{2}}+2 \frac{\sigma^{3}}{\tau^{2} n^{6}}-4 \frac{\sigma^{2}}{\tau^{2} n^{4}}\right)$.

Proof Recall that from the fixed point derivation (equation 4.1.1),

$$
\frac{\sigma}{\tau n^{2}}<1
$$

and by assumption

$$
\begin{aligned}
& \sigma, \tau>0 \\
& \sigma, \tau \leq 1
\end{aligned}
$$

Observe that $\Delta(t)$ changes in the following manner:

$$
\begin{aligned}
-(\Delta(t+1)-\Delta(t))= & a(t)-a(t+1) \\
= & a^{2}(t)\left(\frac{\sigma^{2}}{n^{4}}-(1-a(t))^{2} \tau^{2}\right) \\
= & \Delta(t) \tau\left(2 \frac{\sigma}{n^{2}}-\Delta(t) \tau\right)\left(\Delta(t)+1-\frac{\sigma}{\tau n^{2}}\right)^{2} \text { replacing } a(t) \text { with } \delta(t)+\beta \\
= & \Delta(t) \tau\left(-\Delta^{3}(t) \tau+\Delta^{2}(t)\left(4 \frac{\sigma}{n^{2}}-2 \tau\right)+\Delta(t)\left(6 \frac{\sigma}{n^{2}}-5 \frac{\sigma^{2}}{\tau n^{4}}-\tau\right)+\left(2 \frac{\sigma}{n^{2}}+\right.\right. \\
& \left.\left.2 \frac{\sigma^{3}}{\tau^{2} n^{6}}-4 \frac{\sigma^{2}}{\tau n^{4}}\right)\right)
\end{aligned}
$$

Observe that by equation 4.2.2,

$$
-\Delta^{3}(t) \tau<0
$$


Further,

$$
\begin{aligned}
\Delta^{2}(t)\left(4 \frac{\sigma}{n^{2}}-2 \tau\right) & <\Delta^{2}(t) 4 \frac{\sigma}{n^{2}} \\
& \leq 4 \frac{\sigma}{n^{2}} \quad \text { since } 0<\Delta(t) \leq \frac{\sigma}{\tau n^{2}}<1
\end{aligned}
$$

Moreover,

$$
\Delta(t)\left(6 \frac{\sigma}{n^{2}}-5 \frac{\sigma^{2}}{\tau n^{4}}-\tau\right)<0 \text { by equation } 4.2 .1 \text { and } 4.2 .2
$$

However, by the same equations,

$$
\left(2 \frac{\sigma}{n^{2}}+2 \frac{\sigma^{3}}{\tau^{2} n^{6}}-4 \frac{\sigma^{2}}{\tau n^{4}}\right)>0
$$

Hence, using equations, 4.2.4, 4.2.5, 4.2.6 and 4.2.7 in equation 4.2.3, we have

$$
\begin{aligned}
-(\Delta(t+1)-\Delta(t)) & <\Delta(t) \tau\left(\left(2 \frac{\sigma}{n^{2}}+2 \frac{\sigma^{3}}{\tau^{2} n^{6}}-4 \frac{\sigma^{2}}{\tau n^{4}}\right)+4 \frac{\sigma}{n^{2}}\right) \\
& =\Delta(t) \tau\left(6 \frac{\sigma}{n^{2}}+2 \frac{\sigma^{3}}{\tau^{2} n^{6}}-4 \frac{\sigma^{2}}{\tau n^{4}}\right) \\
& <\Delta(t) \quad \text { for } n \geq 3
\end{aligned}
$$

For computing the minimum change in $(-\Delta(t+1)-\Delta(t))$ observe the following:

$$
-\Delta^{3}(t) \tau \geq-\frac{\sigma^{3}}{\tau^{2} n^{6}} \quad \text { since } 0<\Delta(t) \leq \frac{\sigma^{3}}{\tau^{2} n^{6}}
$$

Further,

$$
\begin{aligned}
\Delta^{2}(t)\left(4 \frac{\sigma}{n^{2}}-2 \tau\right) & \geq \Delta^{2}(t)\left(4 \frac{\sigma}{n^{2}}-2\right) \quad \text { for } \tau=1 \\
& =-2 \Delta^{2}(t)\left(1-2 \frac{\sigma}{n^{2}}\right) \\
& \geq-2 \frac{\sigma^{2}}{\tau^{2} n^{4}}\left(1-2 \frac{\sigma}{n^{2}}\right)
\end{aligned}
$$

Recall from equation 4.2.6, $\Delta(t)\left(6 \frac{\sigma}{n^{2}}-5 \frac{\sigma^{2}}{\tau n^{4}}-\tau\right)<0$. Thus,

$$
\Delta(t)\left(6 \frac{\sigma}{n^{2}}-5 \frac{\sigma^{2}}{\tau n^{4}}-\tau\right) \geq \frac{\sigma}{\tau n^{2}}\left(6 \frac{\sigma}{n^{2}}-5 \frac{\sigma^{2}}{\tau n^{4}}-\tau\right) \text { since } \Delta(t) \leq \frac{\sigma}{\tau n^{2}}
$$


Substituting equations $4.2 .9,4.2 .10$ and 4.2 .11 in equation 4.2 .3 we have,

$$
\begin{aligned}
-(\Delta(t+1)-\Delta(t))> & \Delta(t) \tau\left(\frac{-\sigma^{3}}{\tau^{2} n^{6}}+\frac{\sigma^{2}}{n^{4} \tau^{2}}\left(4 \frac{\sigma}{n^{2}}-2\right)+\frac{\sigma}{\tau n^{2}}\left(6 \frac{\sigma}{n^{2}}-5 \frac{\sigma^{2}}{\tau n^{4}}-\tau\right)+\right. \\
& \left.\left(2 \frac{\sigma}{n^{2}}+2 \frac{\sigma^{3}}{\tau^{2} n^{6}}-4 \frac{\sigma^{2}}{\tau^{2} n^{4}}\right)\right) \\
= & \Delta(t) \tau \frac{\sigma}{n^{2}} \\
< & \Delta(t)
\end{aligned}
$$

Hence $-(\Delta(t+1)-\Delta(t))$, the amount subtracted from $\Delta(t)$ in the next time step, is between $\tau \frac{\sigma}{n^{2}} \Delta(t)$ and $\tau\left(6 \frac{\sigma}{n^{2}}+2 \frac{\sigma^{3}}{\tau^{2} n^{6}}-4 \frac{\sigma^{2}}{\tau^{2} n^{4}}\right) \Delta(t)$, indicating a stepwise contraction in the value of $-(\Delta(t+$ 1) $-\Delta(t))$. This implies that $\Delta(t)$ decays exponentially in $t$.

In particular, $\left(c_{1}\right)^{t} \Delta(0)<t<\left(c_{2}\right)^{t} \Delta(0)$ where $\Delta(0)=1-\beta, \beta$ being the equilibrium value of $a$ and $c_{1}=1-\tau \frac{\sigma}{n^{2}}$ and $c_{2}=1-\tau\left(6 \frac{\sigma}{n^{2}}+2 \frac{\sigma^{3}}{\tau^{2} n^{6}}-4 \frac{\sigma^{2}}{\tau^{2} n^{4}}\right)$. Hence the time taken $t$ to reach $\epsilon$ distance from equilibrium is given by $\frac{\log \frac{1-\beta}{\epsilon}}{\log \frac{1}{c_{2}}}<t<\frac{\log \frac{1-\beta}{\epsilon}}{\log \frac{1}{c_{1}}}$.

Corollary 4.2.1 The Markov Chain corresponding to reversible $n \times n$ completely addressable square assembly is rapidly mixing.

Proof Follows from Theorem 4.2.1 and Theorem 3.2.1.

\subsection{Uniqueness of Equilibrium}

Theorem 4.3.1 The reversible assembly of an addressable square reaches a unique equilibrium for $n \geq 2$.

Proof Let $a_{i, j}(t)$ denote the fraction of $T_{i, j}$ that are free on the right (same variable for free at the top) at time step $t$. Further let $a_{i, j}^{\prime}=a_{i, j}(t+1)$ and $a_{i, j}=a_{i, j}(t)$. Further as before $a$ is the common value of $a_{i, j}$ and $a_{k, l}$ and $a_{m i n}$ denotes the minimum value of $a$ and $a_{\text {max }}$ denotes the maximum value of $a$. Moreover,

$$
1-\frac{\sigma}{\tau n^{2}} \leq a_{i, j}, a_{i, j}^{\prime}, a_{k, l}, a_{k, l}^{\prime} \leq 1
$$

Then for two tiles $T_{i, j}$ and $T_{k, l}$, by substituting equation 4.1.1 in $a_{i, j}^{\prime}$ and $a_{k, l}^{\prime}$ we have

$$
\begin{aligned}
a_{i, j}^{\prime}-a_{k, l}^{\prime}= & \left(a_{i, j}-a_{k, l}\right)\left(1-\frac{\sigma^{2}}{n^{4}}\left(a_{i, j}+a_{k, l}\right)+\tau^{2}\left(a_{i, j}+a_{k, l}\right)+\tau^{2}\left(a_{i, j}^{2}+a_{k, l}^{2}\right)\left(a_{i, j}+a_{k, l}\right)\right. \\
& \left.-2 \tau^{2}\left(a_{i, j}^{2}+a_{i, j} a_{k, l}+a_{k, l}^{2}\right)\right)
\end{aligned}
$$

Observe that, in equation 4.3.2,

$$
\begin{array}{r}
\left.\tau^{2}\left(a_{i, j}+a_{k, l}\right)+\tau^{2}\left(a_{i, j}^{2}+a_{k, l}^{2}\right)\left(a_{i, j}+a_{k, l}\right)-2 \tau^{2}\left(a_{i, j}^{2}+a_{i, j} a_{k, l}+a_{k, l}^{2}\right)\right) \leq 0 \quad \text { since } \\
\quad a_{\max }=1(4.3 .3)
\end{array}
$$


Further,

$$
\begin{aligned}
\tau^{2}\left(a_{i, j}+a_{k, l}\right)+\tau^{2}\left(a_{i, j}^{2}+a_{k, l}^{2}\right)\left(a_{i, j}+a_{k, l}\right) & \\
\left.-2 \tau^{2}\left(a_{i, j}^{2}+a_{i, j} a_{k, l}+a_{k, l}^{2}\right)\right) \geq & -2 \frac{\sigma \tau}{n^{2}}\left(1-\frac{\sigma}{\tau n^{2}}\right)\left(1-2 \frac{\sigma}{\tau n^{2}}\right) \\
& \text { since } a_{\min }=1-\frac{\sigma}{\tau n^{2}}
\end{aligned}
$$

Using equation 4.3.4 in equation 4.3.2,

$$
\begin{aligned}
a_{i, j}^{\prime}-a_{k, l}^{\prime} & \leq\left(a_{i, j}-a_{k, l}\right)\left(1-\frac{\sigma^{2}}{n^{4}}\left(a_{i, j}^{\prime}+a_{k, l}^{\prime}\right)\right) \\
& \leq\left(a_{i, j}-a_{k, l}\right)\left(1-\frac{\sigma^{2}}{n^{4}}\left(2-\frac{\sigma^{2}}{n^{4}}\right)\right) \text { since } a_{\text {min }}=1-\frac{\sigma}{\tau n^{2}}
\end{aligned}
$$

Using equation 4.3.4 again in equation 4.3.2,

$$
\begin{aligned}
a_{i, j}^{\prime}-a_{k, l}^{\prime} & \geq\left(a_{i, j}-a_{k, l}\right)\left(1-\frac{\sigma^{2}}{n^{4}}\left(a_{i, j}^{\prime}+a_{k, l}^{\prime}\right)-2 \frac{\sigma \tau}{n^{2}}\left(1-\frac{\sigma}{\tau n^{2}}\right)\left(1-2 \frac{\sigma}{\tau n^{2}}\right)\right) \\
& \geq\left(a_{i, j}-a_{k, l}\right)\left(1-\frac{2 \sigma^{2}}{n^{4}}-2 \frac{\sigma \tau}{n^{2}}\left(1-\frac{\sigma}{\tau n^{2}}\right)\left(1-2 \frac{\sigma}{\tau n^{2}}\right)\right)
\end{aligned}
$$

Thus $\left|a_{i, j}^{\prime}-a_{k, l}^{\prime}\right|$ is multiplied by a constant factor less than 1 in each time step. This property is true for all $i, j, k, l \in\{1,2, \ldots, n\}$ since $T_{i, j}$ and $T_{k, l}$ are arbitrary tiles. Thus the concentrations of all tiles will go to nearly $a$ "rapidly". By theorem 4.1.1 $a$ evolves to a unique equilibrium value. Further the system eventually attains bond pair independence. Hence the system achieves a unique equilibrium.

\section{2D Periodic Assembly}

The class of periodic assemblies lies between the classes of completely addressable and algorithmic assembly. Consider the simple example of a finite-sized, periodic assembly where the largest unique block is a $n \times n$ addressable square (the finite size constraint is enforced by the size of the preassembled boundary). Note that the only difference between the assembly described in section 4 and this one is that the largest assembly in the former is simply a $n \times n$ square while the size of the largest assembly in the latter case can potentially be infinite (e.g. an assembly of repeated blocks of $n \times n$ square). Despite this difference, this assembly has the same unique equilibrium and update rule as the $n \times n$ completely addressable square.

Theorem 5.1 The periodic assembly where the largest unique block is a $n \times n$ completely addressable square goes to a unique equilibrium. Additionally let $\Delta(t)$ denote the distance of a $(t)$ (the fraction of tiles that are free to the right or top) from the equilibrium value of a: $a_{\text {eq }}$ i.e. $\Delta(t)=a(t)-a_{e q}$. Then $\Delta(t)$ decays exponentially in $t$. In particular, time taken $t$ to reach $\epsilon$ distance from equilibrium is given by $\frac{\log \frac{1-a_{e q}}{\epsilon}}{\log \frac{1}{c_{2}}}<t<\frac{\log \frac{1-a_{e q}}{\epsilon}}{\log \frac{1}{c_{1}}}$ where $c_{1}=1-\tau \frac{\sigma}{n^{2}}$ and $c_{2}=$ $1-\tau\left(6 \frac{\sigma}{n^{2}}+2 \frac{\sigma^{3}}{\tau^{2} n^{6}}-4 \frac{\sigma^{2}}{\tau^{2} n^{4}}\right)$. 


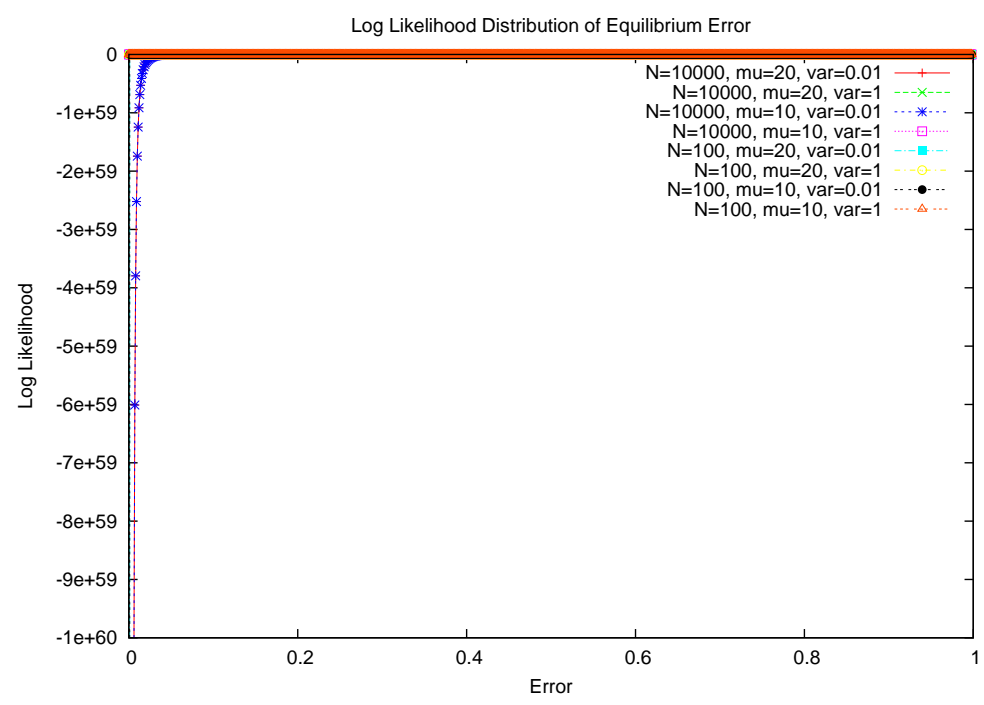

Figure 2: Log likelihood distribution of error at near equilibrium (for reasonable values of aggregate size (N), $G_{s e}$ at equilibrium (mu) and the spread of $G_{s e}$ around equilibrium (var)).

Proof Using the same notation as in Section 4 we observe that the update rule for periodic assembly is exactly the same as 4.1.1. Hence the equilibrium value of $a$ is $\beta=1-\frac{\sigma}{\tau n^{2}}$ Using similar techniques as used in Section 4 we can further prove that the system achieves strong equilibrium. Moreover, theorems 4.2.1 and 4.3.1 directly apply to the periodic assembly.

\section{2D Algorithmic Assembly}

\subsection{Equilibrium Characterization and Convergence Rates}

Unlike completely addressable assembly, algorithmic assembly tile types allow to be reused. By allowing reuse, even a small number of tile types can form complex patterns or perform computations. In other words, in algorithmic assembly tiles of same type might belong to more than one locations. We can handle this mathematically by distinguishing between the tiles of same type that get mapped to different locations in the assembly. Using this mapping we can use the equations from the completely addressable assembly to model algorithmic assembly. We can then add the fractions of these pseudo types to find the fraction of the original tile type. $\mathrm{X}$

\subsection{Error at near equilibrium}

The kinetic DNA tile assembly model (kTAM) by Winfree ${ }^{43}$ states that if algorithmic DNA selfassembly XI achieves equilibrium, the probability of observing a particular assembly $\mathrm{A}$ is given by the Boltzmann distribution

$$
P(A)=\frac{1}{Z} e^{-G(A)}
$$

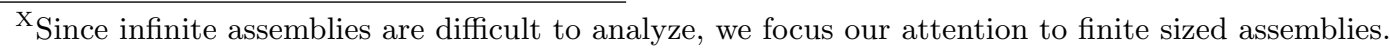

${ }^{\mathrm{XI}}$ We consider only Boolean functions, as in the original aTAM.
} 
where $Z=\Sigma_{A^{\prime}} e^{-G\left(A^{\prime}\right)}$ and $G(A)=n G_{m c}-b G_{s e}$ is the free energy of the assembly. $G_{m c}$ describes the entropic cost associated with tile binding while $G_{s e}$ involves both entropic and enthalpic factors related to the formation of double-helices. Further, if $\eta$ denotes the intrinsic error rate, then

$$
\eta=2 N e^{-G_{s e}}
$$

where $N$ is the size of the assembly.

Let $\epsilon$ be the distance from equilibrium; in the previous sections we saw that the concentration of various subassemblies can be within $\epsilon$ distance from equilibrium in $O\left(\ln \frac{1}{\epsilon}\right)$ time steps. Thus the actual equilibrium $(\epsilon=0)$ is attained in infinite time steps. As the system can only be "close" to equilibrium in finite time, it is interesting to analyze how the error rates vary at near equilibrium. For small $\epsilon$, we define $\epsilon$ distance from equilibrium as the regime in which the perturbation is sufficiently slow and prior work has proved that the free energy distribution around equilibrium for such small scale perturbation is Gaussian. ${ }^{64}$ At $\epsilon$ distance from the equilibrium, we assume that there is one perfect assembly of size $n$ among $2 N+1$ possible choices. Hence the equation for error at equilibrium holds but $G_{s e}$ has changed. Note that $G_{m c}$, being dependent on the concentration of monomers is also different but does not affect the equation for error. $G_{s e}$, representing the free energy, however, has a Gaussian distribution near equilibrium. Hence, using the equation 6.2.2, we can find the distribution of $\eta$ for small linear perturbations from equilibrium.

We can view the above relation as follows: $G_{s e}$ is a random variable with pdf $p_{G_{s e}}$ and if $g$ is a differentiable monotonic invertible function with $\eta=g\left(G_{s e}\right)$ then the probability density function (pdf) of $\eta$ is

$$
p_{\eta}(t)=p_{G_{s e}}\left(g^{-1}(t)\right)\left|\frac{d g^{-1}}{d t}\right|
$$

where $t$ is a free variable. ${ }^{65}$ Here $g\left(G_{s e}\right)=2 N e^{-G_{s e}}$. Hence $g^{-1}(\eta): G_{s e}=\ln \left(\frac{2 N}{\eta}\right)$. Further $\left|\frac{d g^{-1}(\eta)}{d \eta}\right|=\eta^{-1}$. Hence

$$
p_{\eta}=\frac{1}{\sqrt{2 \pi \alpha}} \eta^{-1} e^{-\frac{\left(\ln \left(\frac{2 N}{\eta}\right)-\mu\right)^{2}}{2 \alpha}}
$$

where $\mu$ is the mean of $G_{s e}$ while $\alpha=\operatorname{Var}\left(G_{s e}\right)$ is the variance. Simplifying

$$
p_{\eta}=\frac{e^{-\frac{\mu^{2}}{2 \alpha}}}{\sqrt{2 \pi \alpha}} \eta^{-1}\left(2 N \eta^{-1}\right)^{\mu \alpha^{-1}}\left(0.5 N^{-1} \eta\right)^{\left(\ln \left(2 N \eta^{-1}\right)\right)^{(2 \alpha)^{-1}}}
$$

The probability distribution is very sensitive to the spread of $G_{s e}$ around its mean value (equilibrium value). The log-likelihood of this pdf, however, shows that it is approximately uniform. Hence the probability density function of error around equilibrium is approximately uniform for reasonable values of mean and variance of $G_{s e}$ [Figure 2]. This is acceptable because of the Chebyshev's inequality. Thus for most practical purposes, we can use the error obtained from the Boltzmann 
distribution at equilibrium as error in near equilibrium.

\section{Assembly in Three Dimensions}

\subsection{D Assembly of $n \times n \times n$ Completely Addressable Cube}

One obvious extension of our 2D analysis is an example three dimensional system. The layout of future electronics is predicted to be in three dimensions. For this completely addressable 3D lattices would be very useful.

Theorem 7.1 The assembly of a $n \times n \times n$ completely addressable cube goes to a unique equilibrium. Additionally let $\Delta(t)$ denote the distance of $a(t)$ (the fraction of tiles that are free in the $x, y$ or $z$ direction) from the equilibrium value of $a$ : $a_{e q}$ i.e. $\Delta(t)=a(t)-a_{e q}$. Then $\Delta(t)$ decays exponentially in $t$. In particular, time taken $t$ to reach $\epsilon$ distance from equilibrium is given by $\frac{\log \frac{1-a_{e q}}{\epsilon}}{\log \frac{1}{c_{2}}}<t<\frac{\log \frac{1-a_{e q}}{\epsilon}}{\log \frac{1}{c_{1}}}$ where $c_{1}=1-\left(1-\frac{\sigma}{\tau n^{3}}\right)^{3}\left(\frac{\sigma^{2}}{\tau n^{6}}\right)$ and $c_{2}=1-\frac{3 \sigma^{2}}{\tau n^{6}}$.

Proof This proof here is very similar to the one in Section 4. Suppose that $a_{i, j, k}(t)$ be the fraction of tiles of type $T_{i, j, k}(i, j, k$ denotes the $x, y, z$ coordinates of the tile) that are free in the $x$ direction, $b_{i, j, k}(t)$ be the fraction of tiles of type $T_{i, j, k}$ that are free in the $y$ direction and $c_{i, j, k}(t)$ be the fraction of tiles of type $T_{i, j, k}$ that are free in the $z$ direction. Further assume bond triple equilibrium which is defined similar to bond pair equilibrium in the earlier section. Since we assume same on probability $\sigma$ for all pads in $x, y, z$ direction and same off probability $\tau$ for the same, $a_{i, j, k}(t)=b_{i, j . k}(t)=c_{i, j . k}(t)$. Assume for now that $a_{i j k}$ assumes a common value that is approximately $a$, for all $i, j, k$ 's. We will show later that $a_{i, j, k}$ for all $i, j, k$ 's converge to a common value $a$ exponentially fast.

We estimate the values of $a$ after 1 step. The update rule here is again similar to equation 2.2.20.

$$
a^{\prime}-a=a^{3}(1-a)^{3} \tau^{3}-a^{3} \frac{a^{3}}{a^{3} n^{9}} \sigma^{3}
$$

Observe that this transformation has a unique fixed point in the interval $[0,1]$ given by $\beta=$ $1-\frac{\sigma}{\tau n^{3}}$. In other words when $a=\beta$, the system is in bond pair equilibrium and each $T_{i j k}$ is free in each of the $x, y$ and $z$ direction with an independent probability $\beta$ respectively. Hence, by Theorem 2.2.1, this implies that the tile system has a strong equilibrium.

Further as stated in the theorem, $\Delta(t)$ denotes the distance of $a(t)$ from $\beta$ i.e. $a(t)=\Delta(t)+\beta$. We now prove that $\Delta(t)$ decays exponentially in $t$. For the following derivation it is useful to remember that

$$
\begin{aligned}
0<\sigma, \tau & \leq 1 \\
0<\frac{\sigma}{\tau n^{3}} & <1 \\
n & \geq 2
\end{aligned}
$$


Observe that,

$$
\begin{aligned}
-(\Delta(t+1)-\Delta(t)) & =a^{3}\left(\frac{\sigma^{3}}{n^{9}}-(1-a)^{3} \tau^{3}\right) \\
& =\left(\Delta(t)+1-\frac{\sigma}{\tau n^{3}}\right)^{3}\left(\frac{\sigma^{3}}{n^{9}}-\left(\frac{\sigma}{\tau n^{3}}-\Delta(t)\right)^{3} \tau^{3}\right) \\
& =\Delta(t) \tau\left(\Delta(t)+1-\frac{\sigma}{\tau n^{3}}\right)^{3}\left(\Delta(t)^{2}-3 \Delta(t) \frac{\sigma}{\tau n^{3}}+3 \frac{\sigma^{2}}{\tau^{2} n^{6}}\right)
\end{aligned}
$$

Observe that in equation 7.1 .3

$$
\begin{aligned}
-(\Delta(t+1)-\Delta(t)) & >\Delta(t) \tau\left(1-\frac{\sigma}{\tau n^{3}}\right)^{3}\left(\frac{\sigma^{2}}{\tau^{2} n^{6}}\right) \\
& =\Delta(t)\left(1-\frac{\sigma}{\tau n^{3}}\right)^{3}\left(\frac{\sigma^{2}}{\tau n^{6}}\right) \\
& <\Delta(t) \text { from equation } 7.1 .2
\end{aligned}
$$

Further observe that in equation 7.1.3

$$
\begin{aligned}
-(\Delta(t+1)-\Delta(t)) & <\Delta(t) \tau \frac{3 \sigma^{2}}{\tau^{2} n^{6}} \\
& =\Delta(t) \frac{3 \sigma^{2}}{\tau n^{6}} \\
& <\Delta(t) \quad \text { from equation } 7.1 .2
\end{aligned}
$$

Thus there is a stepwise contraction in $\Delta(t)$. Hence $\Delta(t)$ decays exponentially in $t$. In particular, time taken $t$ to reach $\epsilon$ distance from equilibrium is given by $\frac{\log \frac{1-a_{e q}}{\epsilon}}{\log \frac{1}{c_{2}}}<t<\frac{\log \frac{1-a_{e q}}{\epsilon}}{\log \frac{1}{c_{1}}}$ where $c_{1}=1-\left(1-\frac{\sigma}{\tau n^{3}}\right)^{3}\left(\frac{\sigma^{2}}{\tau n^{6}}\right)$ and $c_{2}=1-\frac{3 \sigma^{2}}{\tau n^{6}}$.

Now we show that all $a_{i, k, j}$ for all $i, j, k$ converge to a common value. Let $a_{i, j, k}(t)$ denote the fraction of $T_{i, j, k}$ that are free on the right (same variable for free at the top) at time step $t$. Further let $a_{i, j, k}^{\prime}=a_{i, j, k}(t+1)$ and $a_{l, m, n}=a_{l, m, n}(t)$. Further as before $a$ is the common value of $a_{i, j, k}$ and $a_{l, m, n}$ and $a_{\text {min }}$ denotes the minimum value of $a$ and $a_{m a x}$ denotes the maximum value of $a$. Further recall that

$$
1-\frac{\sigma}{\tau n^{3}} \leq a_{i, k, j}, a_{i, j, k}^{\prime}, a_{l, m, n}, a_{l, m, n}^{\prime} \leq 1
$$


Then for two tiles $T_{i, j, k}$ and $T_{l, m, n}$, by substituting equation 7.1.1 in $a_{i, j}^{\prime}$ and $a_{k, l}^{\prime}$ we have

$$
\begin{aligned}
a_{i, j, k}^{\prime}-a_{l, m, n}^{\prime}= & \left(a_{i, j, k}-a_{l, m, n}\right)-\frac{\sigma^{3}}{n^{9}}\left(\left(a_{i, j, k}\right)^{3}-\left(a_{l, m, n}\right)^{3}\right) \\
& +\tau^{3}\left(\left(a_{i, j, k}\right)^{3}\left(1-\left(a_{i, j, k}\right)\right)^{3}-\left(a_{l, m, n}\right)^{3}\left(1-\left(a_{l, m, n}\right)\right)^{3}\right) \\
= & \left(a_{i, j, k}-a_{l, m, n}\right)\left(1-\frac{\sigma^{3}}{n^{9}}\left(\left(a_{i, j, k}\right)^{2}+\left(a_{i, j, k}\right)\left(a_{l, m, n}\right)+\left(a_{l, m, n}\right)^{2}\right)\right. \\
& +\tau^{3}\left(1-a_{i, j, k}-a_{l, m, n}\right)\left(\left(a_{i, j, k}\right)^{2}\left(1-\left(a_{i, j, k}\right)\right)^{2}+\left(a_{l, m, n}\right)^{2}\left(1-\left(a_{l, m, n}\right)\right)^{2}\right. \\
& \left.\left.+\left(a_{i, j, k}\right)\left(1-\left(a_{i, j, k}\right)\right)\left(a_{l, m, n}\right)\left(1-\left(a_{l, m, n}\right)\right)\right)\right)
\end{aligned}
$$

Observe that in equation 7.1.7,

$$
\begin{aligned}
\tau^{3}\left(1-a_{i, j, k}-a_{l, m, n}\right)\left(\left(a_{i, j, k}\right)^{2}\left(1-\left(a_{i, j, k}\right)\right)^{2}+\left(a_{l, m, n}\right)^{2}\left(1-\left(a_{l, m, n}\right)\right)^{2}\right. & \\
\left.+\left(a_{i, j, k}\right)\left(1-\left(a_{i, j, k}\right)\right)\left(a_{l, m, n}\right)\left(1-\left(a_{l, m, n}\right)\right)\right) \leq & 0 \quad \text { since } \\
& a_{\max }=1
\end{aligned}
$$

Further, observe that in equation 7.1.7,

$$
\begin{aligned}
\tau^{3}\left(1-a_{i, j, k}-a_{l, m, n}\right)\left(\left(a_{i, j, k}\right)^{2}(1-\right. & \\
\left.\left(a_{i, j, k}\right)\right)^{2}+\left(a_{l, m, n}\right)^{2}\left(1-\left(a_{l, m, n}\right)\right)^{2} & \\
\left.+\left(a_{i, j, k}\right)\left(1-\left(a_{i, j, k}\right)\right)\left(a_{l, m, n}\right)\left(1-\left(a_{l, m, n}\right)\right)\right) \geq & -\tau^{3}\left(1-2 \frac{\sigma}{\tau n^{3}}\right) 3\left(1-\frac{\sigma}{\tau n^{3}}\right)^{2}\left(\frac{\sigma^{2}}{\tau^{2} n^{6}}\right) \\
= & -3 \frac{\tau \sigma^{2}}{n^{6}}\left(1-\frac{\sigma}{\tau n^{3}}\right)^{2}\left(1-2 \frac{\sigma}{\tau n^{3}}\right) \quad \text { since } \\
& a_{m i n}=1-\frac{\sigma}{\tau n^{3}}
\end{aligned}
$$

Using equation 7.1 .8 in equation 7.1.7,

$$
\begin{aligned}
a_{i, j, k}^{\prime}-a_{l, m, n}^{\prime} & \leq\left(a_{i, j, k}-a_{l, m, n}\right)\left(1-\frac{\sigma^{3}}{n^{9}}\left(\left(a_{i, j, k}\right)^{2}+\left(a_{i, j, k}\right)\left(a_{l, m, n}\right)+\left(a_{l, m, n}\right)^{2}\right)\right) \\
& \leq\left(a_{i, j, k}-a_{l, m, n}\right)\left(1-3\left(1-\frac{\sigma}{n^{3}}\right)^{2} \frac{\sigma^{3}}{n^{9}}\right) \\
& \leq\left(a_{i, j, k}-a_{l, m, n}\right) \text { from equation } 7.1 .2
\end{aligned}
$$


Using equation 7.1.9 in equation 7.1.7,

$$
\begin{aligned}
a_{i, j, k}^{\prime}-a_{l, m, n}^{\prime} \geq & \left(a_{i, j, k}-a_{l, m, n}\left(1-\frac{\sigma^{3}}{n^{9}}\left(\left(a_{i, j, k}\right)^{2}+\left(a_{i, j, k}\right)\left(a_{l, m, n}\right)+\left(a_{l, m, n}\right)^{2}\right)\right)\right. \\
& \left.-3 \frac{\tau \sigma^{2}}{n^{6}}\left(1-\frac{\sigma}{\tau n^{3}}\right)^{2}\left(1-2 \frac{\sigma}{\tau n^{3}}\right)\right) \\
\geq & \left(a_{i, j, k}-a_{l, m, n}\right)\left(1-3 \frac{\sigma^{3}}{n^{9}}-3 \frac{\tau \sigma^{2}}{n^{6}}\left(1-\frac{\sigma}{\tau n^{3}}\right)^{2}\left(1-2 \frac{\sigma}{\tau n^{3}}\right)\right) \\
\leq & \left(a_{i, j, k}-a_{l, m, n}\right) \text { from equation } 7.1 .2
\end{aligned}
$$

Thus for all $i, j, k\left(a_{i, j, k}-a_{l, m, n}\right)$ converge to a common value $a$ in logarithmic number of steps. The theorem as stated then readily follows.

\section{Erroneous Assemblies}

Our analysis thus far does not consider assembly errors (e.g: a tile binding at a growth location where it is not supposed to bind); one might reasonably wonder how the analysis would change if they were considered. The main type of error we are interested here is known as error by partial mismatches. This error occurs when a tile binds where it is not intended to bind. An interesting observation is that even with errors the reversible assemblies converge to equilibrium exponentially fast for certain assembly instances. Since the convergence rates depend on the type of assembly and the assembly model, we cannot make any general claim, but we prove this fact for the cases discussed below. In general, the update rule is more or less the same as before, except for the terms that account for the partial matches with on probability $\sigma^{\prime}$ and off probability $\tau^{\prime}$.

\section{1 n-linear Polymerization with Partial Mismatches}

Consider a reversible $n$-linear polymerization, as described in Section 3 except allowing partial mismatch errors. We assume that the probability of a $T_{i} T_{i+1}$ bond is $\sigma$ if they are adjacent; for all other $n-1$ tiles it is $\sigma^{\prime}$, where $\sigma>\sigma^{\prime}$. Similarly, we define the off probabilities $\tau$ and $\tau^{\prime}$ for perfect and partial matches respectively. In particular the main assumptions are

$$
\begin{aligned}
& 0<\sigma^{\prime}(n-1) \leq \sigma \leq \frac{1}{2} \\
& 0<\tau \leq \tau^{\prime}(n-1) \leq \frac{1}{2}
\end{aligned}
$$

Hence, following a similar analysis as in Section 4, the new update rule for $v_{i}(t)$ where $v_{i}(t)$ is the portion of tile $T_{i}$ which are free to the right is as follows (We will drop the subscript $i$ to denote a common $v$ value for all tiles.)

$$
\begin{aligned}
v(t+1)-v(t)= & \frac{-v^{2}(t) \sigma}{v(t)(n-1)+1}-(n-1) \frac{v^{2}(t) \sigma^{\prime}}{v(t)(n-1)+1} \\
& +(1-v(t)) \tau+(n-1)(1-v(t)) \tau^{\prime}
\end{aligned}
$$


This relation comes from the observation that for every tile $T_{i}$, the probability of a perfect match with a $T_{i+1}$ (which is free on the right) is $\frac{v^{2}(t) \sigma}{v(t)(n-1)+1}$. There are, however, $n-1$ possible ways for a partial match with other tiles. The corresponding total probability is $(n-1) \frac{v^{2}(t) \sigma^{\prime}}{v(t)(n-1)+1}$. The $(1-v) \tau$ and $(n-1)(1-v) \tau^{\prime}$ components are derived from the dissociation probabilities.

Observe that for $v$ on the domain $[0,1]$, this transformation has a unique fixed point $\beta$ which is the positive root of the quadratic equation

$$
\frac{-v^{2}(t) \sigma}{v(t)(n-1)+1}-(n-1) \frac{v^{2}(t) \sigma^{\prime}}{v(t)(n-1)+1}+(1-v(t)) \tau+(n-1)(1-v(t)) \tau^{\prime}=0
$$

At this point the system is in bond equilibrium and each $T_{i}$ is free to the right with independent probability $\beta$. Hence the tile system is in strong equilibrium by Theorem 2.2.1. It can also be shown that each $v_{i}$ converge to a common value and hence the system goes to a unique equilibrium. Furthermore we can prove that the convergence happens exponentially fast using techniques from Section 4. We extend this update rule to higher dimensions in the following section. Equilibrium values and convergence rates can be readily derived from the update rule. We sum up our results in the form of the following theorem:

Theorem 8.1.1 The $n$ linear polymerization with partial mismatches goes to a unique equilibrium. Additionally let $\Delta(t)$ denote the distance of $v(t)$ (the fraction of tiles that are free to the right) from the equilibrium value of $v: v_{e q}$ i.e. $v(t)-v_{e q}=\Delta(t)$. Then $\Delta(t)$ decays exponentially in $t$. In particular, time taken $t$ to reach $\epsilon$ distance from equilibrium is given by $\frac{\log \frac{1-a_{e q}}{\epsilon}}{\log \frac{1}{c_{2}}}<t<\frac{\log \frac{1-a_{e q}}{\epsilon}}{\log \frac{1}{c_{1}}}$ where $c_{1}=1-\tau^{\prime \prime}$ and $c_{2}=1-\frac{1}{2}\left(\tau^{\prime \prime}+\sigma^{\prime \prime}+\sqrt{\tau^{\prime \prime} 2+\tau^{\prime \prime} \sigma^{\prime \prime}}\right)$.

Proof This proof is again similar to ones in the previous sections. Observe that the fixed point for $v(t)$ is

$$
v_{e q}=\frac{-(n-2)+\sqrt{n^{2}+4 \rho}}{2(\rho+n-1)}
$$

where $\rho=\frac{\sigma+\sigma^{\prime}(n-1)}{\tau+\tau^{\prime}(n-1)}$. Further observe that the update rule and hence the fixed point is basically the same as that for error-free linear polymerization as considered by Adleman et al. ${ }^{3}$ with the only difference being that the new on and off probabilities are $\sigma^{\prime \prime}=\sigma+\sigma^{\prime}(n-1)$ and $\tau^{\prime \prime}=\tau+\tau^{\prime}(n-1)$ respectively. Note that

$$
\begin{aligned}
& 0<\sigma^{\prime \prime} \leq 1 \\
& 0<\tau^{\prime \prime} \leq 1
\end{aligned}
$$

Using the same technique for computing the upper and lower bounds of distance from equilibrium as Adleman et al., ${ }^{3}$ it can be shown that

$$
\tau^{\prime \prime} \Delta(t)<-(\Delta(t+1)-\Delta(t))<\frac{1}{2}\left(\tau^{\prime \prime}+\sigma^{\prime \prime}+\sqrt{\tau^{\prime \prime 2}+\tau^{\prime \prime} \sigma^{\prime \prime}}\right) \Delta(t)
$$


Thus the amount subtracted from $\Delta(t)$ in the next time step lies between $\tau^{\prime \prime} \Delta(t)$ and $\frac{1}{2}\left(\tau^{\prime \prime}+\right.$ $\left.\sigma^{\prime \prime}+\sqrt{\tau^{\prime \prime} 2+\tau^{\prime \prime} \sigma^{\prime \prime}}\right) \Delta(t)$. In particular, time taken $t$ to reach $\epsilon$ distance from equilibrium is given by $\frac{\log \frac{1-a_{e q}}{\epsilon}}{\log \frac{1}{c_{2}}}<t<\frac{\log \frac{1-a_{e q}}{\epsilon}}{\log \frac{1}{c_{1}}}$ where $c_{1}=1-\tau^{\prime \prime}$ and $c_{2}=1-\frac{1}{2}\left(\tau^{\prime \prime}+\sigma^{\prime \prime}+\sqrt{\tau^{\prime \prime 2}+\tau^{\prime \prime} \sigma^{\prime \prime}}\right)$.

Further, we can show that starting with a different $a_{i}$ for each tile $T_{i}$, all $a_{i}$ 's rapidly squeeze towards a common value since

$$
\left(1-\tau^{\prime \prime}\right)\left|a_{i}(t)-a_{j}(t)\right| \geq\left|a_{i}(t+1)-a_{j}(t+1)\right| \geq \frac{2}{n}\left|a_{i}(t)-a_{j}(t)\right| \quad \text { for } n \geq 3
$$

Moreover, the system goes to independence as shown in the previous sections. The theorem thus stated readily follows.

\subsection{Higher dimensional Assemblies with Partial Mismatches}

Using the same notation as in Section 4, for equilibrium characterization and convergence rates derivation of a $n \times n$ completely addressable square and $\sigma^{\prime}$ and $\tau^{\prime}$ as on and off probabilities for partial mismatches respectively, we state the update rule for a completely addressable square where partial mismatches are allowed:

$$
a^{\prime}-a=a^{2}(1-a)^{2} \tau^{2}+a^{2}(1-a)^{2}\left(\tau^{\prime}\right)^{2}\left(n^{2}-1\right)^{2}-\frac{a^{2} \sigma^{2}}{n^{4}}-\frac{a^{2}\left(\sigma^{\prime}\right)^{2}\left(n^{2}-1\right)^{2}}{n^{4}}
$$

The main assumptions in this model are

$$
\begin{aligned}
& 0<\tau \leq \tau^{\prime}\left(n^{2}-1\right) \leq \frac{1}{\sqrt{2}} \\
& 0<\sigma^{\prime}\left(n^{2}-1\right) \leq \sigma \leq \frac{1}{\sqrt{2}}
\end{aligned}
$$

Theorem 8.2.1 The assembly of a $n \times n$ completely addressable square with partial mismatches goes to a unique equilibrium. Additionally let $\Delta(t)$ denote the distance of a $(t)$ (the fraction of tiles that are free to the right or top) from the equilibrium value of $a: a_{e q}$ i.e. $a(t)-a_{e q}=\Delta_{a}(t)$. Then $\Delta(t)$ decays exponentially in $t$. In particular, time taken $t$ to reach $\epsilon$ distance from equilibrium is given by $\frac{\log \frac{1-a_{e q}}{\epsilon}}{\log \frac{1}{c_{2}}}<t<\frac{\log \frac{1-a_{e q}}{\epsilon}}{\log \frac{1}{c_{1}}}$ where $c_{1}=1-\left(\tau^{\prime \prime} \frac{\sigma^{\prime \prime}}{n^{2}}\right)$ and $c_{2}=1-\tau^{\prime \prime}\left(6 \frac{\sigma^{\prime \prime}}{n^{2}}+2 \frac{\sigma^{\prime \prime} 3}{\tau^{\prime \prime 2} n^{6}}-4 \frac{\sigma^{\prime \prime 2}}{\tau^{\prime \prime} 2 n^{4}}\right)$, $\tau^{\prime \prime 2}=\tau^{2}+\tau^{\prime 2}\left(n^{2}-1\right)^{2}$ and $\sigma^{\prime \prime 2}=\sigma^{2}+\sigma^{\prime 2}\left(n^{2}-1\right)^{2}$.

Proof Observe that if we make the following substitutions

$$
\begin{gathered}
\tau^{\prime \prime 2}=\tau^{2}+\tau^{\prime 2}\left(n^{2}-1\right)^{2} \\
\sigma^{\prime \prime 2}=\sigma^{2}+\sigma^{\prime 2}\left(n^{2}-1\right)^{2}
\end{gathered}
$$

then the equation 8.2.2 is same as equation 2.2.20 with $\tau$ and $\sigma$ replaced with $\tau^{\prime \prime}$ and $\sigma^{\prime \prime}$. Hence the fixed point in this case is

$$
a_{e q}=1-\frac{\sigma^{\prime \prime}}{\tau^{\prime \prime} n^{2}}
$$


Even the equations for computing bounds on $\Delta(t)$ apply and so does Theorem 4.3.1. Further note that

$$
\begin{array}{r}
0<\sigma^{\prime \prime} \leq 1 \\
0<\tau^{\prime \prime} \leq 1 \\
0<\frac{\sigma^{\prime \prime}}{\tau^{\prime \prime} n^{2}}<1
\end{array}
$$

Hence we simply state the results here without the derivation details.

$$
\left(\tau^{\prime \prime} \frac{\sigma^{\prime \prime}}{n^{2}}\right) \Delta(t)<-(\Delta(t+1)-\Delta(t))<\tau^{\prime \prime}\left(6 \frac{\sigma^{\prime \prime}}{n^{2}}+2 \frac{\sigma^{\prime \prime 3}}{\tau^{\prime \prime 2} n^{6}}-4 \frac{\sigma^{\prime \prime 2}}{\tau^{\prime \prime 2} n^{4}}\right) \Delta(t)
$$

Thus $\Delta(t)$ decays exponentially in $t$. In particular, time taken $t$ to reach $\epsilon$ distance from equilibrium is given by $\frac{\log \frac{1-a_{e q}}{\epsilon}}{\log \frac{1}{c_{2}}}<t<\frac{\log \frac{1-a_{e q}}{\epsilon}}{\log \frac{1}{c_{1}}}$ where $c_{1}=1-\left(\tau^{\prime \prime} \frac{\sigma^{\prime \prime}}{n^{2}}\right)$ and $c_{2}=1-\tau^{\prime \prime}\left(6 \frac{\sigma^{\prime \prime}}{n^{2}}+2 \frac{\sigma^{\prime \prime} 3}{\tau^{\prime \prime 2} n^{6}}-4 \frac{\sigma^{\prime \prime} 2}{\tau^{\prime \prime} 2 n^{4}}\right)$.

Moreover, all $a_{i, j}$ s squeeze towards a common value in logarithmic number of steps since

$$
\begin{array}{r}
\left|a_{i, j}-a_{k, l}\right|\left(1-\frac{\sigma^{\prime \prime 2}}{n^{4}}\left(2-\frac{\sigma^{\prime \prime 2}}{n^{4}}\right)\right) \geq\left|a_{i, j}^{\prime}-a_{k, l}^{\prime}\right| \\
\left|a_{i, j}-a_{k, l}\right|\left(1-\frac{2 \sigma^{\prime \prime 2}}{n^{4}}-2 \frac{\sigma^{\prime \prime} \tau^{\prime \prime}}{n^{2}}\left(1-\frac{\sigma^{\prime \prime}}{\tau^{\prime \prime} n^{2}}\right)\left(1-2 \frac{\sigma^{\prime \prime}}{\tau^{\prime \prime} n^{2}}\right)\right) \leq\left|a_{i, j}^{\prime}-a_{k, l}^{\prime}\right|
\end{array}
$$

The theorem as stated then readily follows.

We now shift our attention to 3D assemblies with partial mismatches.

Theorem 8.2.2 The assembly of a $n \times n \times n$ completely addressable cube with partial mismatches goes to a unique equilibrium. Additionally let $\Delta(t)$ denote the distance of a $(t)$ (the fraction of tiles that are free in the $x, y$ or $z$ direction) from the equilibrium value of $a: a_{e q}$ i.e. $a(t)-a_{e q}=$ $\Delta_{a}(t)$. Then $\Delta(t)$ decays exponentially in $t$. In particular, time taken $t$ to reach $\epsilon$ distance from equilibrium is given by $\frac{\log \frac{1-a_{e q}}{\epsilon}}{\log \frac{1}{c_{2}}}<t<\frac{\log \frac{1-a_{e q}}{\epsilon}}{\log \frac{1}{c_{1}}}$ where $c_{1}=1-\left(1-\frac{\sigma^{\prime \prime}}{\tau^{\prime \prime} n^{3}}\right)^{3}\left(\frac{\sigma^{\prime \prime}}{\tau^{\prime \prime} n^{6}}\right)$ and $c_{2}=1-\frac{3 \sigma^{\prime \prime 2}}{\tau^{\prime \prime} n^{6}}$, $\tau^{\prime \prime} 3=\tau^{3}+\tau^{\prime 3}\left(n^{3}-1\right)^{2}$ and $\sigma^{\prime \prime}=\sigma^{3}+\sigma^{\prime 3}\left(n^{3}-1\right)^{2}$.

Proof The update rule for three dimensional erroneous assemblies is

$$
a^{\prime}-a=a^{3}(1-a)^{3} \tau^{3}+a^{3}(1-a)^{3}\left(\tau^{\prime}\right)^{3}\left(n^{3}-1\right)^{3}-\frac{a^{3} \sigma^{3}}{n^{9}}-\frac{a^{3}\left(\sigma^{\prime}\right)^{3}\left(n^{3}-1\right)^{3}}{n^{9}}
$$

The main assumptions for this model are:

$$
\begin{aligned}
& 0<\tau \leq \tau^{\prime}\left(n^{3}-1\right) \leq \frac{1}{2^{1 / 3}} \\
& 0<\sigma^{\prime}\left(n^{3}-1\right) \leq \sigma \leq \frac{1}{2^{1 / 3}}
\end{aligned}
$$


If we make the following substitutions,

$$
\begin{array}{r}
\tau^{\prime \prime 3}=\tau^{3}+\tau^{\prime 3}\left(n^{3}-1\right)^{3} \\
\sigma^{\prime \prime 3}=\sigma^{3}+\sigma^{\prime 3}\left(n^{3}-1\right)^{3}
\end{array}
$$

equation 8.2 .8 basically becomes the same as equation 7.1.1, with $\tau$ replaced by $\tau^{\prime \prime}$ and $\sigma$ replaced by $\sigma^{\prime \prime}$. Hence the fixed point of this equation,

$$
a_{e q}=1-\frac{\sigma^{\prime \prime}}{\tau^{\prime \prime} n^{3}}
$$

Further we reuse the upper and lower bounds for the contraction of $\Delta(t)$ from Section 7 . From equations 8.2.9 and 8.2.11 we have

$$
\begin{array}{r}
0<\sigma^{\prime \prime} \leq 1 \\
0<\tau^{\prime \prime} \leq 1 \\
0<\frac{\sigma^{\prime \prime}}{\tau^{\prime \prime} n^{3}}<1
\end{array}
$$

Following the same lines of reasoning as in the proof of theorem 7.1, we obtain,

$$
\begin{array}{r}
\Delta(t)\left(1-\frac{\sigma^{\prime \prime}}{\tau^{\prime \prime} n^{3}}\right)^{3}\left(\frac{\sigma^{\prime \prime} 2}{\tau^{\prime \prime} n^{6}}\right)<-(\Delta(t+1)-\Delta(t))<\Delta(t) \frac{3 \sigma^{\prime \prime} 2}{\tau^{\prime \prime} n^{6}} \\
\left(a_{i, j, k}-a_{l, m, n}\right)\left(1-3 \frac{\sigma^{3}}{n^{9}}-3 \frac{\tau \sigma^{2}}{n^{6}}\left(1-\frac{\sigma}{\tau n^{3}}\right)^{2}\left(1-2 \frac{\sigma}{\tau n^{3}}\right)\right) \leq a_{i, j, k}^{\prime}-a_{l, m, n}^{\prime} \\
\left(a_{i, j, k}-a_{l, m, n}\right)\left(1-3\left(1-\frac{\sigma}{n^{3}}\right)^{2} \frac{\sigma^{3}}{n^{9}}\right) \geq a_{i, j, k}^{\prime}-a_{l, m, n}^{\prime}
\end{array}
$$

Thus $a(t)$ converges to a unique equilibrium exponentially fast. In particular, time taken $t$ to reach $\epsilon$ distance from equilibrium is given by $\frac{\log \frac{1-a_{e q}}{\epsilon}}{\log \frac{1}{c_{2}}}<t<\frac{\log \frac{1-a_{e q}}{\epsilon}}{\log \frac{1}{c_{1}}}$ where $c_{1}=1-\left(1-\frac{\sigma^{\prime \prime}}{\tau^{\prime \prime} n^{3}}\right)^{3}\left(\frac{\sigma^{\prime \prime} 2}{\tau^{\prime \prime} n^{6}}\right)$ and $c_{2}=1-\frac{3 \sigma^{\prime \prime 2}}{\tau^{\prime \prime} n^{6}}$.

\section{Discussion and Open Questions}

Inspired by Adleman et al.'s work on modeling reversible linear assemblies, we investigated a natural extension of this model to higher dimensions. Since the general problem is difficult, ${ }^{51}$ we focused on an important subclass of $2 \mathrm{D}$ assembly where assembly occurs only by monomer addition. We studied equilibrium characterization and convergence rates of various $2 \mathrm{D}$ assemblies, such as completely addressable structures, periodic assemblies and computational assemblies. Further, we relaxed our model to allow error due to partial mismatches and hence study equilibrium properties of a more realistic model. Besides error due to partial mismatches, we studied the probability of error in assembled algorithmic structures near equilibrium. We further discussed a simple extension of our model to three dimensions. 
Although in this paper we viewed equilibrium as a fixed point, there can be stochastic fluctuations around equilibrium. For instance, the temperature may not be constant at equilibrium due to fluid flow. One open question is how to characterize equilibrium in this situation. Suppose some state variable has a Gaussian distribution at near equilibrium, it would be interesting to find out at what maximum rate we can perturb the system from equilibrium such that the system remains within the $\epsilon$ ball defined around the equilibrium point.

A major limitation of our model is the assumption of monomer addition. We also assume a constant temperature of 2 and a pre-assembled seed and boundary. Although these are reasonable in many cases and the model is quite powerful (Turing Universal as shown by Winfree ${ }^{2}$ ), it is not the only way by which supertiles form or dissociate. A model that allows for addition and dissociation of larger structures would be ideal. A major concern, however, with allowing supertile addition in the model is that the assumption of independent bond association and dissociation may not hold when two supertiles interact/fall apart. Our hypothesis is that this independence will likely depend on their respective conformation (degrees of freedom).

Allowing supertile addition has other drawbacks as well. There are an exponential number of supertiles (exponential in the size of the final assembly) and hence exponential number of cases to consider while deriving the update rule. An intermediate model is a model we call hierarchical supertile addition where at the lowermost level, only monomers can associate/dissociate. In the next level our smallest unit of addition/dissociation is a dimer, followed by a tetramer, octamer, etc. Thus one major open question is how can one characterize the equilibrium and rate of convergence of individual subassemblies to equilibrium in the hierarchical supertile model?

Last but not the least important is the constraint that assembly face always need to be concave. In other words for the ease of analysis we assumed a staircase model which may not be very realistic for several assemblies. Hence our immediate goal is to discard this assumption from the model by estimating equilibrium concentration and convergence rates for a non-monotone assembly growth using conventional techniques for analyzing rapidly mixing Markov Chains (for instance, coupling, conductance).

Acknowledgements: We thank Dana Randall, School of Computing, Georgia Institute of Technology, for bringing the work on the rapid mixing of domino and lozenge tilings to our attention. 


\section{References}

[1] H. Wang, Bell Systems Technical Journal 40, 1 (1961).

[2] E. Winfree, Algorithmic self-assembly of dna, Ph.D. thesis, CalTech (1998).

[3] L. Adleman, Q. Cheng, A. Goel, M. Huang, H. Wasserman, Sixth International Conference on Difference Equations and Applications, 2001.

[4] N. Chelyapov, et al., J. Am. Chem. Soc. 126, 13924 (2004).

[5] T. LaBean, et al., J. Am. Chem. Soc. 122, 1848 (2000).

[6] D. Liu, M. Wang, Z. Deng, R. Walulu, C. Mao, J. Am. Chem. Soc. 126, 2324 (2004).

[7] C. Mao, W. Sun, N. Seeman, J. Am. Chem. Soc. 121, 5437 (1999).

[8] E. Winfree, F. Liu, L. Wenzler, N. Seeman, Nature 394(6693), 539 (1998).

[9] H. Yan, T. LaBean, L. Feng, J. Reif, Proc. Natl. Acad. Sci. USA 100(14), 8103 (2003).

[10] H. Yan, S. Park, G. Finkelstein, J. Reif, T. LaBean, Science 301(5641), 1882 (2003).

[11] J. Xu, T. H. LaBean, S. Craig, DNA Structures are their Applications in Nanotechnology (CRC Press -Taylor \& Francis Group, Boca Raton, 2005).

[12] J. H. Reif, Biomolecular Computing 20, 217 (2002).

[13] R. Bruinsma, W. Gelbart, D. Reguera, J. Rudnick, R. Zandi, Phys. Rev. Lett. 90(24):248101 (2003).

[14] P. Rothemund, Proc. Natl. Acad. Sci. USA 97(3), 984 (2000).

[15] K. Wang, K. Böhringer, J. of Microelectromechanical Systems 16, 140 (2007).

$[16]$.

[17] R. Robinson, Inventiones Math 12, 177 (1971).

[18] B. Grunbaum, G. Shepard (1986), chap. 11.

[19] E. Winfree, X. Yang, N. Seeman, DNA Based Computers II (1999), vol. 44 of DIMACS, pp. $191-213$.

[20] P. Rothemund, E. Winfree, STOC (ACM Press, 2000), pp. 459-468.

[21] L. Adleman, Q. Cheng, A. Goel, M. Huang, STOC (ACM Press, 2001), pp. 740-748.

[22] G. Aggarwal, M. Goldwasser, M. Kao, R. Schweller, SODA (ACM Press, 2004), pp. 880-889. 
[23] E. Winfree, R. Bekbolatov, DNA 9, LNCS 2943, 126 (2003).

[24] L. Adleman, et al., STOC (ACM Press, 2002), pp. 23-32.

[25] L. Adleman, J. Kari, L. Kari, D. Reishus, FOCS (2002), pp. 530-537.

[26] H. Chen, Q. Cheng, A. Goel, M. Huang, P. de Espanes, SODA (2004), pp. 890-899.

[27] Q. Cheng, P. de Espanes, Tech. Rep. 03-793, USC (2003).

[28] Q. Cheng, A. Goel, P. Moisset, FNANO (2004).

[29] M. Cook, P. Rothemund, E. Winfree, DNA9 (2004), vol. 2943 of LNCS, pp. 91-107.

[30] K. Fujibayashi, S. Murata, DNA 10 LNCS pp. 113-127 (2005).

[31] E. Klavins, FNANO (Snowbird, UT, 2004).

[32] E. Klavins, R. Ghrist, D. Lipsky, ICRA (2004).

[33] M. Lagoudakis, T. LaBean, DNA Based Computers V (2000), vol. 54 of DIMACS, pp. 141154.

[34] J. Reif, S. Sahu, P. Yin, DNA10 (2005), vol. 3384 of LNCS, pp. 293-307.

[35] J. Reif, S. Sahu, P. Yin, DNA11, LNCS 3892, 257 (2006).

[36] P. Rothemund, Theory and experiments in algorithmic self-assembly, Ph.D. thesis, University of Southern California (2001).

[37] P. Sa-Ardyen, N. Jonoska, N. Seeman, LNCS 2568, 1 (2003).

[38] R. Schulman, S. Lee, N. Papadakis, E. Winfree, DNA9 (2004), vol. 2943 of LNCS, pp. 108-125.

[39] R. Schulman, E. Winfree, DNA10 (2005), vol. 3384 of LNCS, pp. 319-328.

[40] D. Soloveichik, E. Winfree, DNA11 (2006), vol. 3892 of LNCS, pp. 305-324.

[41] D. Soloveichik, E. Winfree, DNA10 (2005), vol. 3384 of LNCS, pp. 344-354.

[42] E. Winfree, Nanotechnology: Science and Computation (2006).

[43] E. Winfree, Simulation of computing by self-assembly, Tech. Rep. 1998.22, Caltech (1998).

[44] M. Solc, React. Kinet. Catal. lett 28, 17 (1985).

[45] M. O. Magnasco, Phy. Rev. Lett. 78, 1190 (1997).

[46] D. McQuarrie, J. of Applied Probability 4, 413 (1967).

[47] A. Cifferi, Macromol. Rapid Commun. 23, 511 (2002). 
[48] J. N. Israelachvili, Intermolecular and Surface Forces (Academic Press London, 1992).

[49] V. Percec, Science 313, 55 (2006).

[50] G. Ecrolani, J. of Phys. Chem. 107, 5052 (2003).

[51] D. Welsh, Prob. Methods for Algo. Disc. Math. (1998).

[52] E. Guggenheim, Mixtures (Clarendon Press, Oxford, 1952).

[53] E. Cohen, J. D. Boer, Z. Salsburg, Physica XXI, 137 (1955).

[54] J. K. Roberts, Proceedings of the Royal Society of London A (1935), vol. 152, pp. 464-480.

[55] R. Kannan, M. W. Mahoney, R. Montenegro, ISSAC 2003, LNCS 2906, 663 (2003).

[56] D. J. A. Welsh, Disorder in Physical Systems (1990).

[57] M. Mihail, P. Winkler, SODA (1992), pp. 138-145.

[58] A. Berretti, A. Sokal, J. of Statistical Physics 40, 483 (1985).

[59] D. Randall, A. J. Sinclair, SODA (ACM Press, 1994), pp. 593-602.

[60] D. Randall, Computing in Science and Engineering (2006).

[61] D. Randall, SODA (2006), pp. 870-879.

[62] N. Bhatnagar, D. Randall, SODA (2004), pp. 478-487.

[63] D. R. M Luby, A. Sinclair, SIAM J on Computing 31, 167 (2002).

[64] J. Gore, F. Ritort, C. Bustamante, PNAS 100, 12564 (2003).

[65] M. Lavine, Introduction to Statistical Thought (http://www.stat.duke.edu/ michael/book.html, 2006). 